Florida International University FIU Digital Commons

\title{
Attentional Competition: Weapon Focus, Encoding Time, and Memory Accuracy Correlations between Crime Scene Items
}

Seyram Kekessie

Florida International University, skeke001@fiu.edu

DOI: $10.25148 /$ etd.FIDC000090

Follow this and additional works at: https:// digitalcommons.fiu.edu/etd

Part of the Experimental Analysis of Behavior Commons, Other Psychology Commons, and the Social Psychology Commons

\section{Recommended Citation}

Kekessie, Seyram, "Attentional Competition: Weapon Focus, Encoding Time, and Memory Accuracy Correlations between Crime Scene Items" (2015). FIU Electronic Theses and Dissertations. 2209.

https://digitalcommons.fiu.edu/etd/2209 


\title{
FLORIDA INTERNATIONAL UNIVERSITY
}

\author{
Miami, Florida
}

\begin{abstract}
ATTENTIONAL COMPETITION: WEAPON FOCUS, ENCODING TIME, AND MEMORY ACCURACY CORRELATIONS BETWEEN CRIME SCENE ITEMS
\end{abstract}

A dissertation submitted in partial fulfillment of

the requirements for the degree of

DOCTOR OF PHILOSOPHY

in

PSYCHOLOGY

by

Seyram Mawuko Kekessie 
To: Dean Michael Heithaus

College of Arts of Sciences

This dissertation, written by Seyram Mawuko Kekessie, and entitled Attentional Competition: Weapon Focus, Encoding Time, and Memory Accuracy Correlations between Crime Scene Items, having been approved in respect to style and intellectual content, is referred to you for judgment.

We have read this dissertation and recommend that it be approved.

Lindsay Malloy

Ronald P. Fisher

Albert Wuaku

Steve Charman, Major Professor

Date of Defense: April 27, 2015

This dissertation of Seyram Mawuko Kekessie is approved.

$\begin{array}{r}\begin{array}{r}\text { Dean Michael Heithaus } \\ \text { College of Arts and Sciences }\end{array} \\ \hline \begin{array}{l}\text { Dean Lakshmi N. Reddi } \\ \text { University Graduate School }\end{array}\end{array}$

Florida International University, 2015 


\section{DEDICATION}

I dedicate this dissertation to my maternal grandmother, Esther Violet Leh. That I am at the finish line with the flag waving is in no small part due to her patience, nurturing, unconditional support, and prayers.

Finally, this dissertation is dedicated to the memories of my loving paternal grandmother, Ellen Kekessie, and my brothers, Mawusi Dei-Tutu and Norvisi Atsu. They would have been proud. 


\section{ACKNOWLEDGMENTS}

First, I wish to thank my mother, Kafui Ayer, whose unconditional love has remained my source of strength over the years. I would also like to thank my siblings, cousins and my entire family for their constant encouragement throughout this endeavor. Deserving special mention are Kodia Ayer, Kafui Dei-Tutu and Seth Kekessie, whose love and support has been pivotal to my success. I am blessed and grateful to have them in my corner.

I would also like to thank the numerous friends who have over the years proofread my work, provided a listening ear in tough times, celebrated the little milestones, and encouraged me to never stop pushing. Deserving special mention are Flossy Azu, Nana Antwi Boasiako, Kwesi Fynn, Akua Peprah, and Pokua Asafu-Adjei.

I also wish to thank my committee members, Ron Fisher, Lindsay Malloy and Albert Wuaku, for their support, constructive criticism, and patience throughout this process. Their guidance and support was valuable beyond measure.

I would also like to thank my dedicated undergraduate research assistants that made completion of this project possible. Special thanks to Maribel Castro and Martha Armengol. Their help made this process smooth, efficient, and enjoyable.

Finally, I wish to thank my major advisor, Steve Charman, for supporting me from the very beginning of my graduate academic career. His guidance and unwavering belief in my abilities allowed me to successfully complete this project. It would not have been successful without him. 


\begin{abstract}
OF THE DISSERTATION
ATTENTIONAL COMPETITION: WEAPON FOCUS, ENCODING TIME, AND MEMORY ACCURACY CORRELATIONS BETWEEN CRIME SCENE ITEMS
\end{abstract}

\author{
by \\ Seyram Mawuko Kekessie \\ Florida International University, 2015 \\ Miami, Florida \\ Professor Steve Charman, Major Professor
}

The present study develops a theoretical model of attention to examine the relationships between memory for faces and memory for objects within an eyewitness context. 713 participants watched an image that was either displayed for five seconds or twenty seconds, and either included a weapon or no weapon. Subsequently, they were asked to give descriptions of what they saw before viewing a series of person and object lineups that either included the targets or not. Generally consistent with the proposed attentional model, several results were found: (a) witnesses' description accuracy of the crime scene had little or no predictive abilities with regards to their facial identification accuracy; (b) a weapon focus effect was found for faces but not for objects; (c) this weapon focus effect was eliminated among witnesses who had long encoding times; and (d) increased encoding time improved recognition of objects, but not faces. Results suggest that prior inaccuracy on one aspect of testimony is not necessarily indicative of subsequent inaccuracy on another aspect of testimony. The findings advance a model of attention that is able to account for various disparate empirical findings. 


\section{TABLE OF CONTENTS}

CHAPTER

PAGE

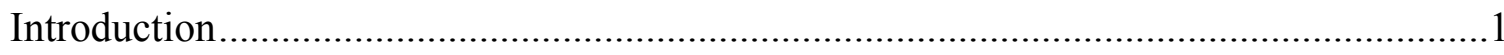

Recall and Recognition Memory in the Eyewitness Literature ...................................4

Memory Processing for Faces versus Non-facial Objects ......................................... 10

Influence of Encoding Time................................................................................... 14

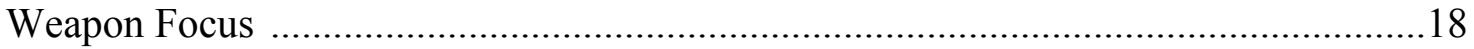

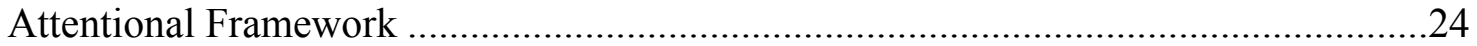

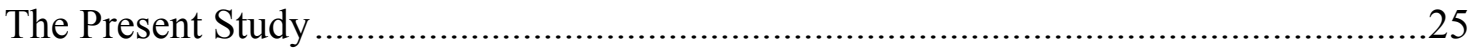

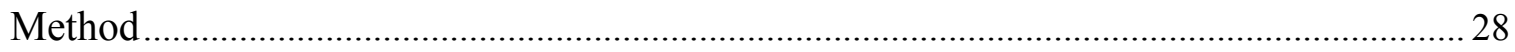

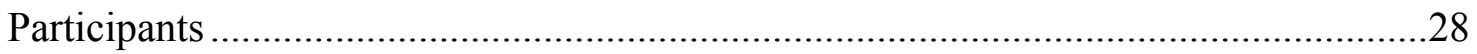

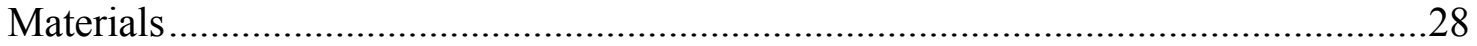

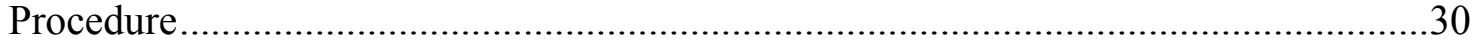

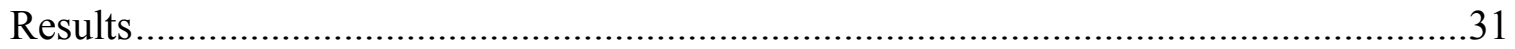

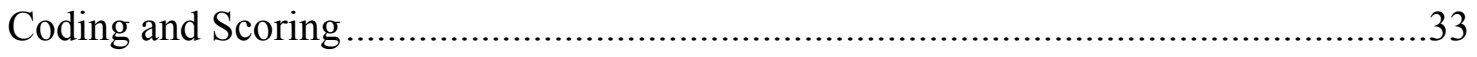

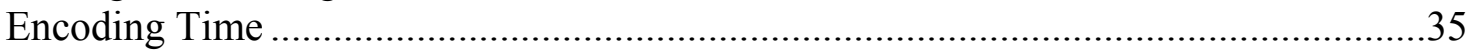

Predicting Witnesses' Lineup Identification Accuracy from their Descriptions ..........37

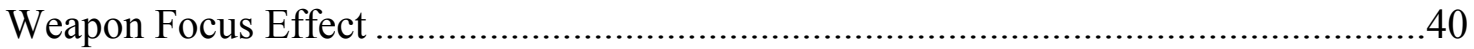

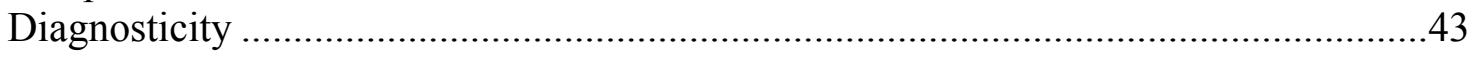

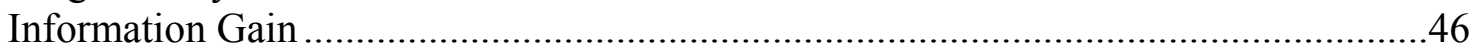

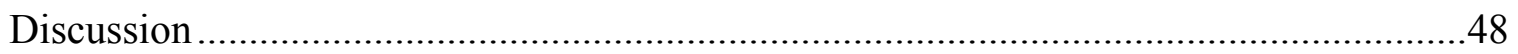

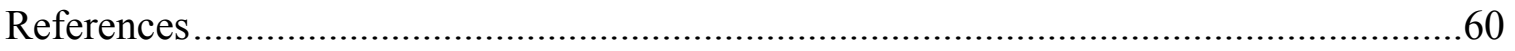

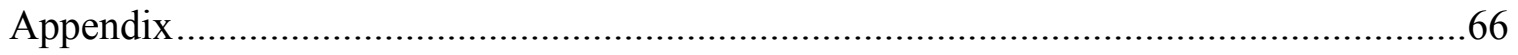

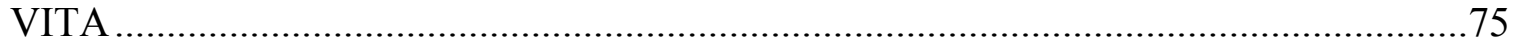




\section{Introduction}

Consider the story of Steve, who decided one afternoon on his way home to purchase some snacks from a convenience store in his neighborhood. He paid for his items and proceeded to the exit, just as another man made his way into the store. Upon entering, the man ordered the store clerk at gunpoint to hand over all the money in the cash register. The store clerk obliged and emptied the contents of the cash register. The man then rushed out of the store and into a waiting car, which fled the scene. Soon afterwards, the police arrived at the scene and began investigating. Based on information gathered from witnesses, the police were able to arrest a suspect a few days later. Steve was one of the witnesses interviewed by the police and he was called to the witness box to testify in court. During cross-examination, the defense attorney asked him if he recalled what he told the police about the color of the vehicle the suspect used to escape. He responded that it was a green car. At this point, the defense attorney introduced security camera footage evidence showing the car in question. Scrutiny of the footage revealed that the vehicle was in fact blue, and not green as indicated by Steve to the police and the court. Does Steve's inaccuracy about the color of the vehicle render him unreliable as a witness? Does his inaccuracy mean that other parts of his testimony cannot be trusted? Is his credibility as a witness undermined? These are all questions that the present study will examine. The objectives of the present study are threefold: (a) to examine the relationships between recognition and recall accuracy of faces, and recognition and recall accuracy of objects; (b) to examine whether these relationships are influenced by the amount of time available to a witness for encoding information; and (c) 
to examine the influence of the presence of a weapon on memory processing and accuracy. To address these questions, a novel theoretical model of eyewitness attention is proposed that ties together these research questions.

A key tenet of an ideal legal system is to render fair and accurate decisions regarding the guilt or innocence of the people who stand accused before a court. Consequently, one of the most important roles of a judge or a juror is to determine whether or not a court witness is being truthful. Indeed, this role is a core component in assessing the credibility of a witness, which may ultimately influence the judge or juror's final verdict (Porter \& ten Brinke, 2009).

During a trial, an attorney's main goal is ultimately to persuade the jury or judge to accept his or her version of the facts (Dial \& Ellis, 2010). One way this can be done is through the testimony of witnesses, which entails calling a witness to the stand, through whom the attorney's theory of the case is presented. Another tactic entails attacking or undermining the credibility of an opposing witness via cross-examination. One of the most common ways a witness' credibility can be undermined during cross-examination is by exposing prior inconsistent or inaccurate information, and using that as a basis to disparage the value of any subsequent information provided by the witness. Indeed, it is common practice for an attorney during cross-examination to for instance retort to a witness: "since you were inaccurate about the color of the suspect's getaway vehicle, why should we believe your facial identification of the suspect in a lineup?" There is ample evidence to suggest that this tactic has been quite effective in many cases (Dial \& Ellis, 2010). At issue is not necessarily whether the tactic is effective, but rather whether it is 
true. More specifically, are inaccuracies with respect to recall memory for objects related to inaccurate facial lineup identifications?

Previous literature suggests that we have a default tendency to believe a witness is accurate and truthful unless there is a compelling reason as to why the witness' testimony should not be trusted (Fisher, Vrij \& Leins, 2010; Gilbert, 1991). In instances where we do not have adequate and relevant knowledge about an event, we tend to rely on factors such as the witness's confidence and the consistency of his or her statements as indicators of reliability (Fisher, Vrij \& Leins, 2010). In some instances however, there is adequate relevant background knowledge about an event, which allows us to objectively assess the accuracy or veracity of a witness' account. For instance, the security camera footage from a crime scene can help investigators objectively ascertain the accuracy of a witness' recollection of a particular crime scene detail. Therefore, a witness who identifies the color of a get-away vehicle as green can be judged to be inaccurate if the security video footage shows that the car was in fact blue.

For the purposes of the present study, I examine the relationship between accuracy on one set of items (i.e., non-facial object information) and the accuracy on another set of items (i.e., facial identification information). In simple terms, does recall or recognition accuracy on non-face items predict facial identification accuracy? Additionally, accuracy may be influenced by the retrieval memory processes involved in recall and recognition. 


\section{Recall and Recognition Memory in the Eyewitness Literature}

Our ability to remember events, people, or objects relies in large part on the mental processes of recall and recognition. Although quite inter-dependent under certain circumstances, the processes of recall and recognition are different at a basic level. Whereas recall demands the retrieval of specific, contextual details of a past encounter, recognition relies more on a subjective sense of previously encountered details of a past encounter (Hollingworth, 1913).

A number of studies have compared recall and recognition memory performance on varying tasks. For instance, some prior studies (e.g., Freund, Brelsford, \& Atkinson, 1969) have shown that when examining scores, recognition memory can be generally superior to recall memory. The superiority of recognition over recall is assumed to be due to the differences in the processes during the time of retrieval (Loftus, 1971).

Specifically, the probability of guessing the right answer is usually higher in recognition than it is with recall. Indeed, it is plausible that the type of information needed for recognition versus recall means that there are different control processes involved for recall and recognition. For instance, with regards to recognition, it is likely possible to generate a correct response with very minimal information about the memory or the target (Loftus, 1971). For example, if the subject can simply remember that the answer to a given question is the name of an animal, s/he is likely to guess correctly if presented with the response option "table" and asked to respond "yes or no." This strategy of course fails when asked to simply recall the answer. 
Some studies have however shown that there are certain circumstances under which recall can be superior to recognition. Specifically, storage processing differences may account for differences in recognition-recall performance. In one study, Loftus (1971) randomly assigned participants to four conditions prior to being given either a recall or recognition test. In one condition, participants were presented with stimulus material to study, and were told that they would later be tested on their memory for that material with a recall task. In another condition, participants were presented with the stimulus material and told that they would later be tested on their memory for that material with a recognition test. In the third condition, participants were presented with the stimulus material and were only told they would be tested, without being told the specific type of test they would be given. Finally, in the fourth condition, participants were given no information about being tested. Results indicated that subjects who knew they would be tested performed better on recall but worse on recognition than those who were not told they would be tested. Loftus interpreted these results as support for the assertion that storage processing differences account for differences in recall-recognition performance.

\section{Recall versus Recognition within an Eyewitness Context}

During crime investigations, investigators routinely interview eyewitnesses to gather details about the crime, and to generate leads that can result in the apprehension of a suspect. These interviews are typically only useful to the extent that the witness can reliably recollect details related to the crime from memory. The recollection of crime details are driven in large part by the memory processes of recall and recognition. For 
instance, a witness's initial description of the perpetrator would rely on the process of recall, whereas a witness's identification of the perpetrator from a lineup would rely on the process of recognition (Ellis, 1984; Tversky, 1973). Thus, the role of recall and recognition processes cannot be overstated in terms of importance to the work of law enforcement officials.

Researchers on eyewitness memory have over the years examined the influence of recall and recognition processes on eyewitnesses' ability to provide accurate accounts of witnessed events. For instance, a plethora of previous research has attempted to examine the relationship between verbal descriptions of suspects and facial recognition (Chance \& Goldstein, 1976; Goldstein \& Chance, 1971; Meissner, Brigham, \& Kelley, 2001; Pigott \& Brigham, 1985; Wells, 1985). Indeed, it is standard procedure for police investigators to request a thorough description of a crime perpetrator shortly after a crime, in order to assist in their investigation to identify and apprehend the suspect. Much of the research on this topic has focused on examining whether verbal descriptions of a perpetrator provided by a witness (a product of recall) can predict subsequent identification of a suspect from a lineup (a product of recognition).

Much of the earlier research on the description-identification relationship revealed that the accuracy with which a face was described was not related to the accuracy of facial identification. For instance, Pigott and Brigham (1985) briefly exposed participants to a target person whose physical characteristics they were later asked to describe. They were then subsequently asked to identify the target person from a lineup in which he was either present or not present. The results showed no relationship between description 
accuracy and identification accuracy. In another study by Chance and Goldstein (1976), subjects who were asked to describe something about a face that would aid them in recognizing it later were not significantly more accurate in identification performance, compared to those who only looked at the faces or who generated word associates. Several subsequent studies supported the view that people who were superior at describing faces were not significantly superior at identifying those faces (e.g., Goldstein, Johnson, \& Chance, 1979; Wolfskiel \& Brigham, 1985).

However, other studies have in fact found a relationship between description accuracy and subsequent identification accuracy. Wells (1985) postulated that the aforementioned studies failed to find a relationship between prior description and subsequent identification for methodological reasons; these studies tended to use only a single stimulus face, as they were designed to test solely whether people who are good describers are also good at identification. According to Wells, however, some faces are easier to describe and easier to identify than others; if so, then we would expect a description-identification relationship to appear when multiple target faces were used as stimuli. Each of the participants in Wells' study viewed one of 88 different target faces in one of two profiles (either straight-on pose or three-quarters profile). Following a filler task, they were asked to describe the face, after which they were given another filler task and were subsequently asked to identify each of the faces among sets of photographs that either contained the original faces or not. The results showed a significant correlation between witnesses' description accuracy and their identification accuracy. Wells 
explained his results as suggesting that faces that are better described are also better identified.

Other studies in the eyewitness literature have taken another approach by examining the legal community's general belief that inconsistent testimony is diagnostic of inaccuracy (Fisher \& Cutler, 1995; Fisher, Vrij, \& Leins, 2010; Gilbert \& Fisher, 2006). The specific aim of these studies was to empirically examine the relationship between consistency and accuracy of witnesses' memory reports in a context where accuracy could be objectively assessed. In simple terms, are inconsistent witnesses any less accurate than consistent witnesses? For instance, Fisher and Cutler (1995) conducted experiments which employed the following basic procedure: One or more confederates intruded and interrupted an ongoing class and stole an object of value. The incident lasted between $30-150$ seconds, after which participants (student-witnesses) were initially asked to describe the intruder via a questionnaire or with an interactive interview format (Day 1). Several days later (Day 2), witnesses were asked again for a description of the intruder. Immediately after giving a second description, participants were shown lineups and asked to identify the intruder. The results showed that inconsistencies in the description of the perpetrator between Day 1 and Day 2 were not related to description accuracy or to lineup identification accuracy, nor was there any sizable correlation between description accuracy and identification accuracy.

Using a different paradigm, Brewer, Potter, Fisher, Bond, and Luszcz (1999) showed participants a video clip of a robbery crime scene and informed them that they were to be subsequently interviewed as witnesses to the crime depicted in the video. 
After viewing the video, the participants completed an hour-long filler activity. The participants were immediately interviewed in either a free-response or forced-response format for the first time after completing the activity. Two weeks later, they were interviewed for the second time, again in either a free-response or forced-response format. The authors showed that overall consistency across interviews was not predictive of overall accuracy. In addition, the accuracy of statements with regards to a specific dimension of testimony (e.g., the suspect's appearance) was not related to accuracy with regards to another dimension of testimony (e.g., the suspect's actions).

The aforementioned studies reveal two important findings that are relevant for the purposes of the present study: (a) there was no correlation between consistency and accuracy; and more importantly (b) there was no correlation between accuracy on one dimension of testimony and accuracy on another dimension of accuracy. More particularly, suspect description accuracy was not related to suspect identification accuracy. A question left unaddressed by these studies, however, is whether the accuracy of witnesses' descriptions for objects is related to their later facial identification accuracy. Thus, one of the principal aims of the present study is to examine whether there is a relationship between object description accuracy and facial description accuracy. In other words, would a witness's accuracy/inaccuracy on memory for objects present at the crime scene (for instance a get-away vehicle) be related to the witness's accuracy/inaccuracy on facial identification?

These prior findings suggest that there would be little correlation between description of objects and facial identification, especially if recall is an independent 
process from recognition. It is however recognized that faces are processed differently than objects; in particular, people are poor at describing faces (Tanaka \& Farah, 1993). This could result in a lack of variability in face descriptions across witnesses, accounting for small description-identification correlations. If, however, people are generally better at describing objects (thus increasing variability across participants in their ability to describe objects), we may expect to find a significant relationship between object description and face recognition. And given that attorneys often discredit witnesses' facial identifications by pointing out inaccuracies in their description of objects, this question also has strong applied value.

\section{Memory Processing for Faces versus Non-facial Objects}

Researchers in the face processing literature often claim that people generally spend a greater amount of time looking at faces than at other stimuli; while there is still some contention as to whether innate face processing exists, developmental research exploring this topic has suggested the existence of a special cognitive processing system for faces that begins during infancy (Pascalis \& Kelly, 2009). For instance, a number of studies have demonstrated that newborn infants show a preference for human faces over other types of visual stimuli (e.g., Johnson, Dziuarawiec, Ellis, \& Morton, 1991). Additionally, from an evolutionary perspective, an organism's survival may depend on its ability to process faces in order to differentiate between in-group and out-group members, and to detect potential threats. In fact, evidence shows that most species are capable of face processing at an early stage of evolution, given that this ability is fundamental to their survival (Pascalis \& Kelly, 2009). Furthermore, for the purposes of 
sexual reproduction, species rely on their ability to individuate different faces when choosing potential mating partners. Therefore, evolution suggests that people may be oriented primarily towards faces, compared to other stimuli.

It is necessary to examine the issue of memory processing for faces and objects within the context of memory research. Most crime scenes are composed of multiple types of details that require encoding by witnesses. For instance, an armed robbery crime scene may involve information about the suspect's identity as well as other non-suspect related information such as the color of nearby houses or cars, or even a weapon. This raises a question as to whether the face of the suspect in this situation would be visually processed in a different manner than the objects would. In fact, a plethora of studies have argued in favor of the uniqueness of faces because they are recognized using visual processing mechanisms that are different from those involved in recognizing other objects (Reed, Stone, Bozova, \& Tanaka, 2003).

At the basic level, facial recognition involves holistic or configural processing of facial features, which simply means that we process and recognize faces as a unitary percept of the components or features of the face (Reynolds \& Pezdek, 1992). In other words, the individual features of a face and the relations between the features are relatively inseparable in terms of how they are processed (Curby \& Gauthier, 2007). Face recognition relies on holistic processing for the purposes of maximizing sensitivity to configural information that constitute a face (Farah et al., 1998). For instance, memory for a face part has been shown to be much better in the context of the original whole face, than when presented alone, a phenomenon referred to as the part-whole effect (Tanaka \& 
Farah, 1993). Also, according to the composite effect (Young et al., 1987), aligning the top half of the face of one individual with the bottom half of the face of another individual makes it difficult to correctly identify the top half (McKone \& Robbins, 2011). In comparison, object recognition is parts-based, where people are able to process and recognize individual parts of an object (Bruce \& Humphreys, 1994; McKone \& Robbins, 2011; Tovee, 1998). Biederman (1987) argued that objects are recognized based on the nature and relative location of their parts, without necessarily encoding the location of the parts in relation to the main axis of the object (Bruce \& Humphreys, 1994).

Indeed, holistic processing has long been considered central to accurate face recognition. Nonetheless, only a few studies have been able to show a direct empirical link between holistic processing and face-recognition ability, with most of the support for the relationship mainly being indirect (Richler, Cheung, \& Gauthier, 2011). In an exception to this trend, Richler, Cheung, and Gauthier examined the relationship between holistic processing and face-recognition by testing participants' face-recognition abilities on the Cambridge Face Memory Test and on a face-identification task. They showed that holistic processing predicted face-recognition ability on both the Cambridge Face Memory Test and on the identification task.

The idea that faces are processed differently than non-face objects has also been suggested by several behavioral studies that have examined the inversion effect, which has over the years proven to be one of the most significantly robust phenomena in the face recognition and object recognition literature (Carey \& Diamond, 1994; Reed et al., 2003; Yin, 1969). Research has shown that faces, compared to objects, are recognized 
more quickly and accurately in their upright position, but are disproportionally impaired by inversion (Carey, 1992; Reed et al., 2003; Yarmey, 1971; Yin, 1969). One explanation offered for the face-inversion effect is that inversion disrupts configural processing. Considering the importance of the role of configuration of facial features in facial recognition, this disruption may account for faces being disproportionately more difficult to recognize when inverted than other objects (Farah, Tanaka, \& Drain, 1995; Leder \& Bruce, 2000; Reed et. al., 2003). In addition, neuroimaging evidence has shown that brain regions that are activated by inverted faces are different from the regions activated by upright faces, and similar to the regions activated by non-facial objects (Haxby et al., 1999; Reed et al., 2003). This is further indication that inversion impairs the normal processing of faces. Additionally, fMRI studies have shown that some face selective brain cell areas can respond three times more strongly to within-class discrimination of faces than to discrimination of objects such as cars and houses (Kanwisher \& Yovel, 2006; McKone \& Robbins, 2011).

Research has not only shown the existence of inversion effects, but has also found these effects to be far more stable for faces than for non-faces. Although inversion effects for object identification decreases with repeated trials, large inversion effects remain for faces even after multiple viewing practice (McKone et al., 2001; McKone \& Grenfell, 1999). In effect, holistic processing for upside-down faces is virtually impossible even after many repeated learning trials (McKone \& Robbins, 2011; Robbins \& McKone, 2003). 
The research discussed so far has outlined both direct and indirect evidence confirming holistic processing for faces, and a lack thereof for individual-level recognition of objects. Also, the developmental and evolutionary research discussed suggests that people are oriented towards faces in a way that they are not for other stimuli. Taken altogether, the evidence suggests that memory processes for recognizing faces are different from the processes involved in recognizing objects. In light of this difference in processing, it is unclear whether face recognition and object recall are associated with one another. In the context of the present study, this means that the processes involved in remembering the face of a suspect would differ from the processes involved in remembering objects he carried, or the car in which he fled. Nonetheless, there is little empirical evidence that speaks to whether a witness' performance on identifying the face of the suspect can be predicted from his or her ability to recall objects from the crime scene.

\section{Influence of Encoding Time}

Some basic memory research studies that have compared memory performance for faces and non-face objects suggest that encoding time is an important variable to consider when examining these relationships. (e.g., Eng, Chen, \& Jiang, 2006; Wheeler \& Treisman, 2002). For instance, Tversky and Sherman (1975) presented students with 60 slides containing images of different objects such as a television, teakettle, etc. Each participant was randomly assigned to one of four groups, which determined whether they viewed each image for $0.25,0.50,1.00$, or 2.00 seconds. After the presentation of the 60 images, participants were then given a test of free recall, and were subsequently asked to 
participate in a recognition test, in which pairs of images (each stimulus and a distractor) were presented and the participants were required to indicate which of the images they originally viewed. In line with the authors' predictions, the results showed that the recall and recognition of the images generally improved as exposure time increased.

Although Tversky and Sherman (1975) found results showing that encoding time significantly improved recognition of visual items, their stimulus consisted of only images of objects. However, a number of studies have specifically examined the effect of exposure time on the recognition of facial images. In one such study, Ellis, Davies, and Shepherd (1977) presented participants with thirty facial images and randomly assigned them to one of five exposure duration conditions. Participants viewed the image for 0.25 , $0.50,1.00,2.00$, or 4.00 seconds. They were then given a recognition test that included the addition of thirty new facial images that acted as distractors. Similar to the results found in studies that used images of objects, participants' recognition performance improved as the exposure duration increased. A later meta-analysis (Shapiro \& Penrod, 1986) on 128 eyewitness identification and face recognition studies confirmed this finding: increased exposure duration reliably improved recognition for faces. Taken altogether, these studies examining the influence of exposure duration on recognition clearly indicate that the amount of time a witness is able to view a face can be an important predictor of identification accuracy. Additionally, the results suggest that the improvement in recognition as exposure duration increases is generalizable across objects, faces, and possibly other visual images. 
A few studies have indirectly examined comparative differences in facial recognition memory performance and object recognition memory performance. These studies typically examine the capacity of the visual short-term memory (VSTM) - which refers to a memory component that aids in the processing and remembering of faces and objects - and the extent to which it is influenced by the complexity of the items stored, and the processing strategy used during encoding. For instance, Curby and Gauthier (2007) examined the extent to which the capacity of the VSTM is influenced by the encoding of faces versus objects. Curby and Gauthier asked participants to study up to five faces and objects (e.g., watches and cars) displayed on a screen. Participants were randomly assigned to view the faces and objects for varying lengths of time ( $0.50 \mathrm{vs} .1 .50$ vs. 2.50 vs. 4 seconds). They were subsequently given a recognition test where they were presented with a single face and were tasked with deciding whether that face was part of the initial faces they viewed. A similar recognition test was administered for the objects. According to the results, when participants studied the display image for half a second, more objects were stored in VSTM than faces. However, when exposure duration increased to 4 seconds, an advantage for faces emerged, such that VSTM capacity for faces exceeded that for objects. This advantage was specific only to faces encoded in the upright orientation, as those encoded upside-down showed no such advantage. According to the researchers, the interaction of VSTM capacity and face orientation suggests that the advantage for face VSTM when there is adequate encoding time is due to holistic processing. 
According to Curby and Gauthier (2007), the greater increase in VSTM capacity for faces than objects as encoding time increased, may be explained by the differences in complexity between faces and objects. Specifically, more time was needed to encode faces because they are greater in complexity than objects. This is consistent with findings from previous research suggesting that objects of greater complexity require more space in VSTM than those of lesser complexity (Eng et al., 2006). Previous studies have also shown slower visual search rates for faces compared to other objects, which suggests that faces, compared to objects, place a greater burden on encoding mechanisms (Curby \& Gauthier, 2007; Eng et al., 2006). Therefore, when encoding time is limited, complex objects require more time to be encoded into VSTM than less complex objects. With sufficient encoding time, however, the influence of complexity on VSTM capacity is reduced due to the availability of adequate time for perceptual processing.

The differential impact of encoding time on object and face memory suggests that the relationship between object and face memory will change as a function of encoding time. It is important to note as well that many of the past studies focus on memory for a single item at a time (e.g., Curby \& Gauthier, 2007; Ellis, Davies \& Sherpherd, 1977). However, actual eyewitnesses in real life situations are exposed to multiple items simultaneously within a scene. The influence of encoding time when witnesses are exposed to multiple items in a scene could be especially important since there will be multiple items necessitating encoding. How might the relationship between object and face memory be affected by exposure time? One possibility is that there would be a tradeoff between object and face memory at low encoding times but not at higher encoding 
times. Essentially, greater memory for one item would come at the expense of memory for the other item when encoding time is limited. Based on results from Curby and Gauthier's (2007) study, I predict that there would be an attentional trade-off between object and face memory at short encoding times. In more specific terms, this would for instance mean that higher recall accuracy on objects should predict lower accuracy on facial identifications. This is due to the complex nature of faces, which may require more encoding time, and therefore may draw attention from the object when there is limited encoding time. When encoding time increases to the point where complex faces can be encoded substantially and thus attention diverted elsewhere, that negative correlation should lessen, or may even become positive. If this prediction is true, it means that we should be able to predict a witness' performance accuracy on facial lineups from his or her performance accuracy on objects, but only when there is adequate encoding time.

The objects involved in the mock crime used in the present study include a bag, a car, and a weapon. However, much eyewitness research has shown that weapons may constitute a special class of objects, whose mere presence may impact memory processing of details in a way that the other objects may not. As such, memory for weapons is discussed separately.

\section{Weapon Focus}

A large number of crimes typically involve the use of a weapon either as an injury-inflicting tool or as a threat against a victim (Maass \& Kohnken, 1989). Over the years, an increasing number of studies have examined one of the most central characteristics of a crime, namely, the presence of a weapon. Most of the research in this 
field has directly examined the tendency for the presence of a weapon to impair a witness' ability to subsequently identify a perpetrator, a phenomenon referred to as the weapon focus effect (Steblay, 1992).

There are several reasons why the presence of a weapon could negatively impact recognition accuracy. The majority of the studies in the weapon focus literature have proffered two main theories to explain the weapon focus effect. The first is the arousal/threat hypothesis, which relies on Easterbrook's (1959) cue-utilization hypothesis proposing that attention and arousal interact to determine how we utilize aspects of our perceptual environment. According to this hypothesis, arousal decreases the number of cues that we can monitor simultaneously. Consequently, when highly aroused, we would tend to focus more on the central cues in our environment, to the detriment of peripheral cues (Fawcett, Russell, Peace, \& Christie, 2013). Applying this idea to weapon focus, the arousal/threat hypothesis suggests that when a weapon is present, it will induce a state of arousal due to its threatening nature. Consequently, people will focus their attention on the source of the arousal, which should lead to heightened memory for central cues such as the weapon, at the expense of memory for peripheral cues such as characteristics of the suspect (Easterbrook, 1959; Yerkes \& Dodson, 1908).

In contrast to the idea that a high state of arousal will lead to attentional narrowing, a second theoretical explanation was proposed based on the idea that it is the unusualness of a weapon that draws attention. According to this novelty/unusual item hypothesis (Loftus \& Mackworth, 1978), a weapon is considered unusual in many contexts and is therefore inconsistent with the individual's mental schema or 
representation of the scene. For instance, people will not commonly associate a gun or knife with a parking lot (versus a shooting range for instance). Therefore, during a gunpoint robbery taking place at a parking lot, a witness is likely to spend attentional resources resolving the conflict existing between the weapon and their mental schema representing the parking lot. This will result in a detrimental effect for memory for peripheral details such as the suspect's face (Fawcett et al., 2013; Loftus \& Mackworth, 1978).

Regardless of the theoretical explanation underlying the effect, several empirical studies assessing the effect of the presence of a weapon on a witness's recognition ability have found support for the weapon focus effect (Cutler, Penrod, \& Martens, 1987; Loftus, Loftus, \& Meso, 1987; Maass \& Kohnken, 1989; Steblay, 1992). For instance, Loftus et al. (1987) presented participants with slides showing a customer interacting with a cashier in a restaurant. Participants viewed a slide that depicted the customer either handing the clerk a check, or pointing a gun at the cashier. Participants were subsequently given a recognition task to identify the target (customer). The results showed that recognition was poorer when there was a weapon involved, compared to when there was a check involved. Importantly, the researchers used a corneal reflection device to track the eye movements of subjects. Researchers recorded both the number as well as the duration of the eye fixations. Their results provided evidence confirming that eye fixation on a weapon distracted from a witness's attention to other relevant details, thereby negatively impacting recognition accuracy. 
In the most direct test of the weapon focus effect, Cutler, Penrod, and Martens (1987) examined the effect of weapon visibility on identification accuracy. In the high weapon visibility condition, a gun was displayed throughout the video footage, whereas in the low weapon visibility condition the weapon remained hidden (in the perpetrator's pocket) throughout the video footage. Participants were then administered a recognition task to identify the perpetrator. Similar to other studies, the results showed a decrease in identification accuracy when there was high weapon visibility.

The aforementioned studies were initially criticized as lacking in generalizability to real crime situations, since the participants in the study were uninvolved observers. According to this view, since the suspect in these studies was presented in slides or video clips, participants in the study were never truly threatened by the presence of a weapon like they would have in real crime situations. Maass and Kohnken's (1989) study addressed this concern by adopting an experimental paradigm that used a syringe as the weapon. The syringe was a clever and useful way of ethically simulating a weapon that most people are likely to find somewhat personally threatening. Participants were approached by an experimenter who either held a syringe or a pen. In both conditions, half of the participants were told they would be administered an injection whereas the other half were given no such information. The authors found that exposure to the syringe significantly decreased subsequent recognition of the target from a lineup.

A meta-analytic study by Steblay (1992) also reviewed twelve different studies that addressed the weapon focus effect and found a significant overall difference between weapon-present and weapon-absent conditions, such that the presence of a weapon led to 
a reduction in identification accuracy. In other studies examining the weapon focus effect, results showed that participants continue to focus on a weapon even when they were explicitly instructed to focus on the target's face (Tooley, Brigham, Maass, \& Bothwell, 1987). Collectively, these results indicate that the weapons focus effect is relatively robust across variations in experimental variables.

As discussed earlier, previous research has established that the weapon focus does reliably occur when there is a visible, threatening weapon, particularly during crimes of short duration. There is, however, no data examining how the weapon focus effect depends on encoding time. If the presence of a weapon draws the witness's attention from the perpetrator, we can predict that the effect of a weapon on facial recognition accuracy will be greater when encoding time is limited. When there is sufficient encoding time, the witness would have adequate time to encode the weapon and possibly encode other details.

The majority of the studies examining the weapon focus effect concentrate mostly on its effect on the recognition accuracy of faces. Consequently, there is a paucity of research that examines how memory accuracy for objects is influenced by the presence of a weapon. Indeed, the few studies that have attempted to expand the examination of the effect to include memory accuracy for objects have only assessed participants' memory of perpetrator characteristics such as clothing and facial features (Steblay, 1992). Hence, it is difficult to determine whether the presence of a weapon could for instance negatively affect a witness's memory for relevant crime scene objects such as the get-away car used by the suspect. One of the goals of the current study is thus to examine whether a weapon 
negatively influences recognition accuracy for non-face objects. For instance, one possibility is that due to the novelty or uniquely arousing nature of the weapon (Maass \& Kohnken, 1989), its presence results in the weapon drawing attention from the other objects.

Prior research has largely established that weapon presence negatively influences identification accuracy. However, previous research is not definitive on whether this negative impact is specific to memory for the perpetrator holding the weapon, or whether it extends to memory for all faces present during the crime. Due to the fact that the present study uses a design involving two perpetrators, there is an opportunity to examine the impact of weapon presence in a multi-perpetrator crime situation. This study presents an opportunity to investigate whether the presence of the gun would negatively influence memory for the face of the co-perpetrator in the crime. Indeed, there is practical value to this since witnesses' ability or inability to identify all the perpetrators involved in a multiperpetrator crime would have implications for whether a criminal is apprehended or left on the streets.

The real world implications of weapon focus is substantial. It is well established that eyewitness testimony is an integral and important aspect many criminal cases, and in some cases an eyewitness's testimony is relied upon to secure a conviction (Fawcett et al., 2013). Based on the findings from the weapon focus literature, the present study was developed to expand upon the weapon focus effect in a number of important and specific ways: (a) to examine whether encoding time moderates the weapon focus effect; (b) to 
examine whether there is a weapon focus effect on memory for objects; and (c) to examine whether weapon focus effect applies to a perpetrator not holding the gun.

\section{Attentional Framework}

The review of past literature led to the development of a theoretical framework which shapes the present study's predictions, and may explain potential findings. This framework, which I term the "attentional competition" framework (ACF), is based on the idea that when people encounter a scenario containing multiple elements, those elements compete with each other for our attentional resources. Due to the limitations of our attentional resources, how we expend those valuable attentional resources would be influenced by various factors, one of which is encoding time. As previously discussed, past literature on memory processing for faces versus objects suggests the likelihood that people are more oriented towards viewing faces, compared to other visual stimuli. Therefore, in a scenario involving people and objects, faces should immediately draw attentional resources, followed by unusual or arousing objects (such as a weapon), finally followed by usual objects. Relating this framework to the present study, the amount of time available to a witness to simultaneously encode details should influence memory performance differentially for faces and objects. If a greater amount of attentional resources are immediately devoted to encoding faces, we should expect there to be heightened memory for face recall or recognition, at the expense of object recall or recognition. This should result in no correlation (or a negative correlation) between object description accuracy and face identification accuracy at short encoding times. However, when there is adequate encoding time available, an individual has sufficient 
time to focus attention on the objects after first encoding face details. Thus, we would expect to see larger description-accuracy correlations for longer encoding times.

Furthermore, according to the $\mathrm{ACF}$, weapons draw attention before other objects do. Consequently, detrimental effects of weapon presence on memory for other details should be greatest when there is limited time available to encode information. When there is limited encoding time, attentional resources may be spent on the weapon, resulting in poor memory for objects at short encoding times. When there is adequate encoding time however, attentional resources can be diverted to non-weapon objects. Therefore, the weapon focus effect should be smaller at long encoding times. Further, because faces and weapons should be immediately capture attention, an increase in encoding time should benefit memory for objects more than memory for faces and weapons. Based on the framework discussed above, I next outline the specific hypotheses of the present study.

\section{The Present Study}

Are there relationships between recognition and recall accuracy of faces, and recognition and recall accuracy of objects? This primary research question is core to the aims of the present study. There are at least two possible ways for such a relationship to occur. First, accuracy on one item (object) may be positively correlated with accuracy on the other item (face), if memory performance on these two items is generally reflective of the underlying quality of a witness's overall memory. Indeed, this viewpoint is fundamental to the legal strategy of undermining a witness's credibility on one item by pointing out his or her prior inaccuracy on another item. Second, accuracy on these two items could be negatively correlated if increased attention for one object (thereby likely 
resulting in greater accuracy) is related to decreased attention for the other (thereby resulting in lesser accuracy). This outcome would essentially rebut the aforementioned legal strategy of undermining a witness' credibility using prior inaccuracy. Based on the attentional competition framework, I hypothesize that the relationship between memory for a face and memory for an object will be dependent on encoding time: when encoding time is limited, the competition for attention among stimuli will result in attention being given to faces (rather than objects), resulting in no (or even a negative) correlation between memory for faces and memory for objects. However, when encoding time is increased, attention can be diverted to objects, thus resulting in a reduced (or eliminated) negative correlation.

Based on the attentional competition framework, I hypothesize that witnesses' facial identification accuracy will be significantly greater when there is no weapon present, compared to when a weapon is present, and that this weapon focus effect will occur for both the person holding the weapon and the person not holding the weapon. Furthermore, according to the ACF, attention will be diverted to weapons before other objects. Thus, I predict that there will be a weapon focus effect on objects when encoding time is limited (and thus there is a competition for attention). However, at longer encoding times, attentional competition is lessened and attention can be diverted to other objects; thus, I predict that the weapon focus effect will be reduced (or even eliminated) at longer encoding times. If this prediction is true, it would imply that the weapon focus effect may be neutralized when there is adequate time available for witnesses to encode information. 
Finally, the design of the present study, which involves both recall and recognition tests for faces and objects, allows us to address a secondary issue: The accuracy of eyewitness identifications from object lineups. A very small handful of studies have examined the utility of non-traditional lineups in addition to the typical facial lineups pervasively adopted by investigators. For instance, Pryke, Lindsay, Dysart, and Dupuis (2004) investigated the use of multiple lineups to identify a target. The authors presented participants with facial, body, voice, and clothing lineups after watching a scene. The results showed that multiple identifications of the suspect by a witness from the different independent lineups were highly diagnostic of the suspect's guilt, suggesting that non-face lineups have diagnostic value. However, this research has examined only items related to the suspect (clothes, voice, or body); there is little to no research examining whether objects not related to the suspect contain diagnostic value. Indeed, there is practical value to examine the usefulness of object lineups. Often times, a crime may involve the use of several objects, some of which may be central to the particular crime. The identities of such objects may be important for the purposes of the work of law enforcement officials. In real cases for instance, it is of enormous benefit if a witness can correctly identify central elements of a crime such as the gun or vehicle used by a perpetrator during the commission of a crime. Further, this study also allows for a comparison of witnesses' object lineup identification accuracy and their object description accuracy. This ultimately allows us to determine whether object lineups provide any beneficial value, which could be useful for the purposes of law enforcement investigations. 
In summation, the hypotheses of the present study, predicted by the attentional competition framework, are as follows: (a) when encoding time is limited, there will be an attentional trade-off between facial encoding and object encoding, resulting in a negative correlation between memory for faces and memory for objects; (b) when encoding time is increased, this negative correlation will be reduced or eliminated; (c) the weapon focus effect will generalize to objects; (d) the weapon focus effect would be moderated by encoding time, such that there will be a negative impact on memory accuracy for faces and objects when encoding time is limited, compared to when there is adequate encoding time; and (e) identifications of objects from object lineups will contain diagnostic value.

\section{Method}

\section{Participants}

Participants in the study consisted of 736 undergraduate students at Florida International University, recruited through SONA systems. Twenty-three participants were excluded from analysis due to incomplete responses, resulting in a final total of 713 participants. All participants received extra credit as compensation for their participation.

\section{Materials}

Stimulus image. The stimulus image is a picture depicting the crime scene, which was created specifically for this study. Using a picture instead of a video ensured that all the objects and faces in the scene were exposed for the same amount of time within the scene. Additionally, using a picture instead of a video also enabled the experimenter to 
control the angle at which the items were captured and presented on screen to the participant. The picture showed the two perpetrators (male and female), a bag, and a vehicle all in one shot. For stimulus sampling purposes (Wells \& Windschitl, 1999), four versions of the stimuli materials were used (weapon vs no weapon; targets A vs targets B). Targets A consisted of two perpetrators, a bag, and a car that differed completely from the ones used as targets B; otherwise the scenes were identical. The weapon was treated as both a dependent variable (tested for recall or recognition as are the other objects) and an independent variable (its presence manipulated). There were no other faces or distinct objects included in the shot in order to ensure that the only faces and objects shown in the picture were that of the perpetrators and objects intended for recall and recognition accuracy testing. The stimulus image was presented on desktop computers and laptops in a laboratory setting. Participants in the short encoding condition were exposed to the image for 5 seconds, whereas those in the long encoding condition were exposed to the image for 20 seconds. This study adopted a basic procedure that is consistent with several previous studies that have used pictures or slides as stimulus materials (e.g., Tooley et al, 1987), and manipulated encoding time using comparable time frames (e.g., Curby \& Gauthier, 2007) prior to testing witnesses' memory.

Lineups. To select the photographs used in constructing the lineups, two people were asked to independently view the stimulus image and provide specific descriptions of the characteristics of each person and object. A list of the most common descriptors for each item was subsequently compiled. Examples of common descriptors for the perpetrators included "male," "dark hair," "Hispanic," "long hair", "tall," and "average 
build" etc. Examples of common descriptors for the objects included "sedan", "black car", "backpack", "bag with a logo", "kitchen knife", and "hand gun" etc. The list of common descriptors was subsequently given to a different set of two people, who looked through a set of twenty photographs for description matches. The seven most selected photographs (including the photograph of the actual perpetrator or target object) were used to construct the lineups. Two types of line-ups were created. Target absent (TA) lineups consisted of six members (fillers), none of which was the perpetrator or target. These fillers matched the description of the perpetrator. The target present (TP) line-up consisted of a six-member line-up, with the perpetrator included as a member of the lineup replacing one of the six fillers. TP and TA lineups involving the objects seen in the video were also created in the same manner as those involving faces. Therefore, for example, a six-item TP lineup included the actual bag seen in the video in addition to five fillers matched to description, whereas the corresponding TA lineup consisted of six bags that were all fillers. Pilot data were collected for the purpose of choosing a designated innocent suspect from the foils in each target absent lineup. Participants in the pilot test viewed the stimulus image and were subsequently asked to select the lineup member they thought was the perpetrator or the target object they saw. The lineup member chosen the most became the designated innocent suspect.

Questionnaire. A questionnaire was created to test participants' recall of the objects and faces seen in the video. Participants were asked both open-recall as well as closed-ended, cued-recall type of questions. In the open-recall questionnaire, participants were given the following instruction: "Describe everything you saw in the photo image 
that you were presented with earlier. Specifically, your task is to describe the information in the photo in a way such that it would aid someone else in identifying the items. Remember to describe all the information in as much detail as possible.' Examples of the cued-recall questions included: 'What is the color of the bag?', 'What is the color of the hair of the male suspect?', 'What is the race of the female suspect?' etc. Responses on the questionnaire were subsequently coded to determine accuracy.

Filler task. Participants were given a filler task that simply involved solving a maze puzzle. This task was timed to last for about a minute. Participants were given the filler task at two points. Each participant was given the first maze immediately after viewing the stimulus picture and before answering the open-ended and cued recall questions. The second maze was presented immediately after answering those questions and prior to completing the lineup identification task.

\section{Procedure}

The study was designed using Qualtrics survey tools and the entire study was completed on a computer. Participation in the study took place in a computer lab at the Modesto Maidique Campus at Florida international University. Participants were randomly assigned to conditions in a 2 (weapon vs no weapon) X 2 (Target A vs Target B) X 2 (encoding time: long vs. short) between subjects design. Participants were also randomly assigned to view target-present and target-absent lineups for each of the lineups. Upon arrival, each participant was assigned a computer on which all stimuli were presented. After signing the consent form and agreeing to participate in the study, each participant was randomly assigned to an experimental condition. The basic procedure, 
which all participants followed, entailed viewing the stimulus image first for either 5 seconds or 20 seconds, depending on condition. These exposure times were chosen based on the exposure times used in previous studies examining memory for single items within a scene (e.g., Curby \& Gauthier, 2007). All participants were then given the first filler task which was a maze puzzle timed to last for approximately one minute. Afterwards, they were given a questionnaire asking them to describe items and faces seen in the image in as much detail as possible. Questions included both open-ended descriptions of the scene as well as directed questions towards specific objects. Participants were then presented with the second filler task, which was also a different maze puzzle timed to last for approximately one minute. Subsequently, they were presented with facial and object lineups for identification decisions. Each randomly assigned participant was given multiple lineups, testing the participant's memory for the two faces, and each of the objects. With regards to the facial lineups, each participant was randomly assigned to either a TP lineup or a TA lineup. Similarly with regards to each of the non-weapon object lineups (i.e., car and bag), all participants were given either a TP lineup or a TA lineup. Those in the weapon condition were randomly assigned to a TP or TA weapon lineup in addition.

Due to the fact that participants are asked to describe the faces and objects before they are asked to identify them in a lineup, there is a concern as to whether the results of the study could be affected by the verbal overshadowing effect, which refers to the detrimental effect of verbal description on subsequent face recognition (Finger \& Pezdek, 1999). Previous research has shown that the verbal overshadowing effect can be reduced 
or even eliminated under some circumstances. For instance, Finger and Pezdek showed that inserting a delay between verbal description and facial identification significantly reduced the verbal overshadowing effect. It is partly for this reason that a filler task was given to participants after the description task and prior to the lineup identification task.

There were four factors manipulated between-subjects. These factors include the target stimuli (Targets A or B), the presence of a weapon (weapon present or weapon absent), stimulus encoding time (short or long), and the nature of the various lineups (TA or TP). In keeping with the procedure used in previous studies, participants were not given specific information about the length of time they will be exposed to the stimulus image. There were vaguely informed that the image "may be displayed for only a few seconds." After providing their recall and lineup identification responses, all witnesses were given a questionnaire assessing other testimony-relevant measures such as their confidence levels. Participants were then debriefed and dismissed.

\section{Results}

\section{Coding and Scoring}

A detailed coding scheme was developed to score the accuracy of participants' responses to the open-ended and cued-recall questions. A unit of information is defined as any piece of information that in and of itself can be useful to an investigator's work. Responses were scored into the following categories: Accurate (any correct unit of information), Inaccurate (any wrong unit of information), Don't remember (responses such as “I can't remember" or "I forgot"), Don't know/Not sure (responses such as "I 
don't know" or I am not sure"), Unscorable (any information of little meaningful value to an investigator, and hence cannot be scored), and Total (total sum of the units of information).

In the accurate category, a score of 1 was awarded for every unit of information that was deemed to be correct. For instance, if the participant stated that "there were two people and a black car," a score of 1 was awarded for "two," "people," "black," and "car" (assuming these responses were correct). The number of accurate units was then summed up, thereby resulting in a total of 4 accurate units for that particular participant. Similarly, in the inaccurate category, a score of 1 was awarded for each inaccurate unit of information provided by the participant, the sum of which was calculated to determine the total number of inaccurate units for a particular participant. For example, if the participant stated that "there were three people and a blue car," then it was scored as 2 inaccurate units, and 2 accurate units. The total number of units of information in this instance would be 4 (the sum total of the accurate plus inaccurate units). A similar scoring protocol was applied in scoring the remaining categories, with 1 point tallied for each "don't remember," “don't know/not sure," or "unscorable" unit.

An overall accuracy rate score for each participant was computed by summing up the number of accurate units, and dividing by the total number of units (accurate + inaccurate). Similarly, the overall inaccuracy rate of a participant was defined as the sum of the total number of inaccurate units, divided by the total number of units. Therefore, if a participant provided 2 correct units of information and 2 inaccurate units of 
information, the accuracy rate would be $2 / 4$ or 0.50 , and the inaccuracy rate would also be $2 / 4$ or 0.50 .

Reliability of scoring was assessed by having two blind independent raters score the responses of 72 participants (approximately 10 percent of the total sample), each of whom had given complete responses to all the questions and were randomly selected across the different conditions. For each participant, the two raters' scores for the responses were compared, and a reliability score was calculated. Across the 72 participants, the interrater reliability score (Cronbach's alpha) was 0.99 for number of accurate units (range $=0.99-1.00), 0.93$ for number of inaccurate units (range $=0.87-$ 1.00 ), 0.80 for number of "Don't remember" responses (range $=0.70-1.00$ ), 0.82 for number of "Don't know/Not sure" responses (range $=0.70-1.00), 0.92$ for number of unscorable units (range $=0.85-1.00$ ), and 0.99 for total number of units (range $=0.99-$ $1.00)$.

\section{Encoding Time}

The following set of analyses focus on examining the influence of encoding time on facial identification accuracy, facial description accuracy, object identification accuracy, and object description accuracy. The various proportions are illustrated in Table 1.

Influence of encoding time on facial identifications. There was no significant

difference in a witness' likelihood of making a correct identification from a target-present lineup when encoding time was 5 seconds $(M=0.42, S D=0.45, N=269)$, compared to 
when encoding time was 20 seconds $(M=0.46, S D=0.46, N=268), t(535)=1.04, p=$ $0.30, d=0.09$.

Among witnesses who viewed a target absent lineup, there was no significant difference in a witness' likelihood of correctly rejecting the lineup when encoding time was 5 seconds $(M=0.49, S D=0.47, N=267)$, compared to when encoding time was 20 seconds $(M=0.56, S D=0.47, N=265), t(530)=1.77, p=0.08, d=0.15$.

Influence of encoding time on object identifications. Witnesses were significantly less likely to make a correct identification of objects when encoding time was 5 seconds $(M=0.51, S D=0.40, N=269)$, compared to when encoding time was 20 seconds $(M=0.71, S D=0.38, N=264), t(531)=5.75, p<.01, d=0.50$.

Witnesses were also significantly less likely to correctly reject the lineup when encoding time was 5 seconds $(M=0.56, S D=0.40, N=272)$, compared to when encoding time was 20 seconds $(M=0.68, S D=0.39, N=269), t(539)=3.75, p<.01, d=$ 0.32 .

Influence of encoding time on facial descriptions. Witnesses' facial description accuracy when they were allowed 5 seconds of encoding time $(M=0.72, S D=0.17, N=$ 359) did not significantly differ from when they were allowed 20 seconds of encoding time $(M=0.74, S D=0.15, N=354), t(711)=1.01, p=0.31, d=0.08$.

Influence of encoding time on object descriptions. Witnesses' object

description accuracy was significantly less when encoding time was 5 seconds $(M=0.69$, 
$S D=0.21, N=359)$, compared to when encoding time was 20 seconds $(M=0.74, S D=$

$0.21, N=354), t(711)=3.20, p<.01, d=0.24$.

Overall, these results show that an increase in encoding time from 5 to 20 seconds had beneficial effects for object identification and object description accuracy, but not for facial identification and facial description accuracy. These results are consistent with the attentional competition framework, which predicts that attention is drawn first to faces and then only subsequently to objects if there is enough time. Thus, the ACF predicts, and current results show, that increased exposure time should primarily benefit object memory as opposed to face memory.

\section{Predicting Witnesses' Lineup Identification Accuracy from their Descriptions}

Overview. Various correlational analyses were performed to examine the relationships between object descriptions, object identifications, facial descriptions, quantity of information, and facial identifications. Since participants saw a different number of target present and target absent lineups due to random assignment, the facial identification accuracy score was calculated separately for target present and target absent lineups by taking the average sum of the witness' accuracy scores on the male and female lineups. First, I examine the correlation between overall description accuracy (accuracy of open-ended recall provided by witness), overall object description accuracy, overall facial description accuracy and facial identification accuracy for both target present and target absent lineups. Subsequently, I examine the correlation between overall description quantity, overall facial description quantity, overall object description quantity and facial identification accuracy. These correlations are illustrated in Table 2. 
Throughout this section, z-tests are used to examine whether differences in encoding time affected the magnitude of these correlations.

Overall description accuracy. Witnesses' overall description accuracy significantly predicted their likelihood of making a correct lineup identification of the targets, $r(535)=0.15, p<.01$. However, encoding time did not moderate this effect, $z=$ $0.24, p=0.81$.

Witnesses' overall description accuracy did not predict their likelihood of correctly rejecting a target-absent lineup, $r(530)=-0.01, p=0.86$. Encoding time did not moderate this effect, $z=-0.11, p=0.91$.

Overall object description accuracy. Witnesses' overall object description accuracy significantly predicted a witness' overall likelihood of making a correct lineup identification of the targets, $r(535)=0.09, p=0.03$; however, encoding time did not moderate this effect, $z=1.29, p=0.19$.

Witnesses' overall object description accuracy did not predict a witness' likelihood of correctly rejecting a target-absent lineup, $r(531)=0.04, p=0.39$; however, encoding time did moderate this effect, $z=0.46, p=0.65$.

Overall facial description accuracy. Witnesses' overall facial description accuracy significantly predicted their likelihood of making a correct lineup identification of the targets, $r(535)=0.08, p=0.05$. Encoding time did not moderate this effect, $z=$ $0.35, p=0.73$. 
Witnesses' overall facial descriptive accuracy did not predict their likelihood to correctly reject a target-absent lineup, $r(530)=0.02, p=0.62$. Encoding time did not moderate this effect, $z=0.34, p=0.73$.

Overall description quantity. The amount of overall descriptive information given significantly predicted a witness's likelihood of making a correct identification, $r(535)=0.13, p<.01$. Encoding time did not moderate this effect, $z=0.35, p=0.73$.

The amount of overall descriptive information significantly predicted a witness's likelihood of correctly rejecting a target-absent lineup, $r(530)=0.10, p=0.02$. Encoding time did not moderate this effect, $z=0.69, p=0.49$.

Facial description quantity. The overall amount of facial descriptors given did not significantly predict a witness's likelihood of making a correct lineup identification of the targets, $r(535)=0.05, p=0.27$. Further analyses showed that encoding time did not moderate this effect, $z=0.35, p=0.73$.

The overall amount of facial descriptors did not significantly predict a witness's likelihood of correctly rejecting a target-absent lineup, $r(530)=0.07, p=0.10$. Encoding time did not moderate this effect, $z=0.23, p=0.82$.

Object description quantity. The amount of overall object descriptive information did not significantly predict a witness's likelihood of making a correct lineup identification of the targets, $r(535)=0.03, p=0.47$. Encoding time did not moderate this effect, $z=0.46, p=0.65$. 
The amount of overall object descriptive information marginally predicted a witness's likelihood of correctly rejecting a target-absent lineup, $r(530)=-0.08, p=0.06$. Encoding time did not moderate this effect, $z=-0.93, p=0.35$.

In summary, the various correlational analyses reveal that description accuracy and quantity had either no predictive abilities for facial identification accuracy, or at best very weak predictive abilities, accounting for approximately $1.5 \%$ of the variance in accuracy, and that this was not moderated by encoding time.

\section{Weapon Focus Effect}

The next set of analyses focus on the weapon focus effect. Specifically I examine whether the presence of a weapon significantly influenced facial identification accuracy, facial description accuracy, object identification accuracy, and object description accuracy. Subsequently, I examine whether the weapon focus effect is influenced by encoding time.

It should be noted that for the purposes of these overall analyses, I collapsed across all target faces, and also collapsed across all target objects in order to examine overall facial description and identification accuracy, as well as overall object description and identification accuracy. Nonetheless, I also present the various identification accuracy proportions separated by each target across weapon presence, encoding time, and lineup type conditions. These proportions are illustrated in Table 3.

Facial lineup identifications. Consistent with the weapon focus effect, witnesses who viewed a target present lineup were significantly more likely to correctly identify the 
target when there was no weapon $(M=0.49, S D=0.47, N=270)$, than when a weapon was present $(M=0.39, S D=0.44, N=267), t(535)=2.50, p=.01, d=0.22$. Further analyses revealed that the size of this weapon focus effect on identification accuracy did not vary significantly based on which of the two targets held the weapon, $t(265)=0.16, p$ $=.91, d=0.02$.

Among witnesses who viewed a target absent lineup, there was no significant difference in correct rejection of the lineup when there was no weapon $(M=0.53, S D=$ $0.47, N=266)$, compared to when a weapon was present $(M=0.53, S D=0.47, N=266)$, $t(530)=0.00, p=1.00, d=0.00$.

Object identifications. There was no significant difference between correct identifications on objects when there was no weapon $(M=0.60, S D=0.40, N=266)$, compared to when a weapon was present $(M=0.62, S D=0.40, N=267), t(531)=0.64, p$ $=.52, d=0.06$.

Among witnesses who viewed a target absent lineup, there was no significant difference between correct rejection of the lineup when there was no weapon $(M=0.61$, $S D=0.39, N=270)$, compared to when a weapon was present $(M=0.63, S D=0.40, N=$ 271), $t(539)=0.42, p=.68, d=0.04$.

Facial descriptions. Witnesses were significantly less accurate in describing the targets' faces when a weapon was present $(M=0.71, S D=0.17, N=362)$, compared to when a weapon was not present $(M=0.75, S D=0.15, N=351), t(711)=3.31, p<.01, d$ $=0.25$. 
Object descriptions: There was no significant difference in object description accuracy when a weapon was present $(M=0.70, S D=0.20, N=362)$, compared to when there was no weapon $(M=0.73, S D=0.21, N=351), t(711)=1.63, p=.10, d=0.12$.

\section{Influence of encoding time on the weapon focus effect. One of the key}

questions the present study examines is whether the amount of time available to a witness to encode information influences the weapon focus effect. As shown in Table 3, the weapon focus effect was found for the Male B target. Therefore, logistic regression analysis was conducted to examine whether the weapon focus effect found for that target from target present lineups was moderated by encoding time. Identification accuracy was regressed onto weapon presence, encoding time, and the interaction between these two variables. The regression model was statistically significant, $\chi^{2}(1)=8.93, p<.01$, and explained $6.6 \%$ of the variance, $R^{2}=.07$. The results showed that there was a marginally significant interaction between encoding time and weapon presence, $\chi^{2}(1)=3.44, p=$ $.06, \beta=1.19$. There was a significant main effect of encoding time, $\chi^{2}(1)=3.76, p=.05$. Generally supporting the hypothesis that the presence of a weapon would have a more negative impact on accuracy when encoding time is limited, compared to when there is adequate encoding time, the results showed that the weapon focus effect occurred when participants viewed the image for 5 seconds, $\chi^{2}(1)=7.69, p<.01$, but was eliminated when they viewed the image for 20 seconds, $\chi^{2}(1)=0.05, p=.83$.

Overall, these results support the firmly established prediction that the presence of a weapon negatively impacts correct identification for faces. This effect occurred regardless of which target held the weapon. Additionally, encoding time moderated this 
effect such that there was a weapon focus effect when participants were allowed 5 seconds of encoding time, but not when they were allowed 20 seconds of encoding time, consistent with predictions from the ACF. According to this model, at short encoding times, the witness's attention is drawn by both the weapon and the faces and he/she must encode the details in a limited amount of time; the weapon competes with the faces for attentional resources, resulting in a weapon focus effect. However, as encoding time increases, this competition decreases and adequate attentional resources can be allocated to both the faces and the weapon, resulting in an elimination of the weapon focus effect. The hypothesis that the weapon focus effect would generalize to objects, however, was not supported.

\section{Diagnosticity}

In addition to standard face lineups, the present study also examined witnesses' accuracy on various object lineups. The present study's integration of object lineups (consisting of car lineups, bag lineups, and weapon lineups) provides the opportunity to examine the extent to which responses to these lineups can provide useful information.

One way to quantify the amount of information contained from a given lineup is to calculate its diagnosticity, which indexes the extent to which a target identification is indicative of that target's guilt or innocence. Specifically, diagnosticity values calculate the likelihood that an identified target is the actual perpetrator, rather than an innocent target (Wells \& Lindsay, 1980). In the context of the present study, diagnosticity estimates similarly provide information about the likelihood that an identified object (car, bag, or weapon) is the actual object the witness saw, rather than a filler object. 
Diagnosticity is especially valuable when comparing lineup procedures to determine superiority in probative value (Wells \& Olson, 2002; Charman \& Wells, 2007). The diagnosticity estimate of a suspect or target identification is calculated as the ratio of the target identification rate from target present lineups to the false alarm rate from target absent lineups. A diagnosticity value of 1 indicates that an identified target is equally likely to be guilty as innocent; as values get larger, a target identification is more likely to indicate the suspect's guilt. Thus, the greater the diagnosticity, the more information a suspect identification provides to a trier of fact. Diagnosticity of target identifications for the various lineups was calculated across encoding time conditions and compared via ztests (see Wells \& Olson, 2002).

Diagnosticity of car identification responses. Among participants who were allowed an encoding time of five seconds, diagnosticity of a car identification was 5.89, meaning that a witness was 5.89 times more likely to correctly identify the target car when it is in the lineup, than to identify a filler when it was not in the lineup. When encoding time increased to twenty seconds, diagnosticity of a car identification increased to 12.5 , meaning that a witness was 12.5 times more likely to correctly identify the target car in a target present lineup, than identify a filler in a target absent lineup.

Subsequent analyses conducted to examine whether encoding time significantly influenced diagnosticity of the car identification revealed that increased encoding time significantly increased the diagnosticity of the car identification, $z=2.9, p<.01$. In other words, a car identification provided more information after a long exposure compared to a short exposure. 
Diagnosticity of bag identification responses. Among participants who were allowed an encoding time of five seconds, diagnosticity of a correct identification was 1.8. When encoding time increased to twenty seconds, diagnosticity of a correct identification increased to 3.75. Subsequent analyses revealed that increased encoding time significantly increased the diagnosticity of the bag identification, $z=2.35, p=.02$.

Diagnosticity of male suspect identification responses. When participants were allowed an encoding time of five seconds, diagnosticity of a correct identification was 3.17. When encoding time increased to twenty seconds, diagnosticity of a correct response increased to 4.89. Subsequent analyses revealed that encoding time did not significantly change the diagnosticity of the identification, $z=1.05, p=.29$.

Diagnosticity of female suspect identification responses. As encoding time increased from five seconds to twenty seconds, diagnosticity of a correct identification increased from 5.5 to 7 . Subsequent analyses revealed that encoding time did not significantly change the diagnosticity of the identification, $z=0.65, p=.52$.

Diagnosticity of weapon identification responses. As encoding time increased from five to twenty seconds, diagnosticity of a correct identification increased from 1.17 to 1.38 respectively. Subsequent analyses revealed that encoding time did not significantly change the diagnosticity of the identification, $z=0.8, p=.42$.

It should be noted that the diagnosticity analyses above were computed by collapsing across the male targets, female targets, bags, cars, and weapons. However, I also calculated diagnosticity estimates separately for each of the targets, as illustrated in 
Table 4. With the exception of one of the male targets, the diagnosticity analyses generally followed the same pattern. Overall, the diagnosticity analyses showed that both object and suspect identifications have diagnostic value, and diagnosticity of the object identifications generally increased with an increase in encoding time. This suggests that the identification responses received from object lineups benefits from an increase in time. This finding is consistent with the attentional competition framework, which predicted that increased exposure time should be particularly likely to result in greater memory for objects compared to faces or weapons, since attention would be initially captured by faces and weapons at short encoding times, and only diverted to objects with further encoding opportunity.

\section{Information Gain Analyses}

In order to determine the extent to which we can update our knowledge about a correct target identification based on witnesses' response, information gain analyses were conducted, which are derived from a Bayesian statistical analysis (see Wells \& Olson, 2002). There are two factors that determine the information gained from a lineup decision. The first is the diagnostic value of the decision (obtained from the diagnosticity ratios reported above), and the second is the likelihood of the suspect's guilt prior to knowing how a witness performed on an identification task (often referred to as the prior probability). Specifically, information gain is determined by examining the difference between the prior probability of guilt and the posterior probability of guilt (i.e., the probability of the suspect's guilt after learning about the witness's identification response). 
Because the prior probability of the suspect's guilt is not known, information gain curves were created that graph the information gained from a lineup identification as a function of the prior probability, consistent with prior research (Charman \& Cahill, 2012; Wells \& Olson, 2002). The amount of information gained was calculated for target identifications for the male, female, car, bag, and weapon targets, and are illustrated in Figure 1, Figure 2, Figure 3, Figure 4, and Figure 5, respectively.

Among witnesses who were allowed 5 seconds of encoding time, information gain associated with the knowledge that a witness correctly identified the male suspect peaked at approximately 0.28 (at a prior probability of approximately 0.35 ). When participants were allowed 20 seconds of encoding time, information gain associated with the knowledge that a witness correctly identified the male suspect peaked at approximately 0.29 (at a prior probability of approximately 0.35 ).

Among witnesses who were allowed 5 seconds of encoding time, information gain associated with the knowledge that a witness correctly identified the female suspect peaked at approximately 0.40 (at a prior probability of approximately 0.30 ). When participants were allowed 20 seconds of encoding time, information gain associated with the knowledge that a witness correctly identified the female suspect peaked at approximately 0.45 (at a prior probability of approximately 0.30 ).

Among witnesses who were allowed 5 seconds of encoding time, information gain associated with the knowledge that a witness correctly identified the car target peaked at approximately 0.42 (at a prior probability of approximately 0.30 ). When participants were allowed 20 seconds of encoding time, information gain associated with 
the knowledge that a witness correctly identified the car target peaked at approximately 0.56 (at a prior probability of approximately 0.20 ).

Among witnesses who were allowed 5 seconds of encoding time, information gain associated with the knowledge that a witness correctly identified the bag target peaked at approximately 0.15 (at a prior probability of approximately 0.45 ). When participants were allowed 20 seconds of encoding time, information gain associated with the knowledge that a witness correctly identified the bag target peaked at approximately 0.32 (at a prior probability of approximately 0.35 ).

Among witnesses who were allowed 5 seconds of encoding time, information gain associated with the knowledge that a witness correctly identified the weapon peaked at approximately 0.04 (at a prior probability of approximately 0.50 ). When participants were allowed 20 seconds of encoding time, information gain associated with the knowledge that a witness correctly identified the weapon peaked at approximately 0.08 (at a prior probability of approximately 0.45 ).

\section{Discussion}

The present study reveals a few main findings. First, the various correlational

analyses showed that overall, the accuracy and quantity of a witness's descriptions of the crime scene had little or no predictive abilities with regards to facial identification accuracy. At best, the results indicated very weak predictive abilities, accounting for approximately $1.5 \%$ of the variance in identification accuracy. This was not moderated by encoding time. Second, the presence of a weapon, as predicted, negatively affected 
facial description accuracy and the correct identification rate on facial lineups. Further, the deleterious effect of the presence of a weapon was limited to face identification and descriptions; the weapon did not negatively affect either object description accuracy or object identification accuracy. Third, there was a moderating effect of encoding time on the weapon focus effect such that the weapon focus effect occurred when participants had limited encoding time, but not when they had adequate encoding time. Fourth, encoding time significantly influenced object description and object identification accuracy, but did not affect facial description and facial identification accuracy. Finally, both object and facial identifications were of diagnostic value; the diagnostic values of object identifications increased with an increase in encoding time, whereas the diagnostic values of face identifications did not significantly increase with an increase in encoding time.

One of the questions this study sought to specifically examine was whether a witness's memory for various stimuli in a crime scene - faces, weapons, and objects were related to one another, and whether any such relationship is influenced by the amount of time available to a witness to encode information. During the course of investigating a crime, witnesses are typically asked to describe objects or environments, and rarely asked to identify them. Similarly, witnesses are usually shown a lineup for the purposes of identifying a perpetrator. While the results generally showed that there was no substantial relationship between facial identification and description accuracy or quantity, a key line of inquiry involved specifically examining the relationship between accuracy on object descriptions and facial identification accuracy. Our results do not provide empirical support for the idea that witnesses' lineup identifications of a target can 
be discredited by their inaccuracy on object descriptions. This is important as a relationship between object description and facial identification is often assumed by attorneys; results of the current study undermine this assumption.

Drawing from the attentional competition framework, it was hypothesized that there would be an attentional trade-off resulting in little (or a negative) correlation between accuracy on objects and accuracy on faces at short encoding times, but that longer encoding times would result in a positive relationship. In actuality, the results showed that witnesses' accuracy on object descriptions did not predict their facial identification accuracy. Further analyses showed that encoding time also had no effect on the relationship.

There are both theoretical and empirical reasons that can explain the lack of a substantial relationship between description accuracy and identification. Logically, whether accuracy on describing objects is correlated with accuracy on identifying a suspect from a lineup depends on the extent to which processing of the two events is independent; if the processing is independent, then there should be little to no relationship (Fisher and Cuervo, 1983; Brewer et al., 1999). For instance, Fisher and Cuervo (1983) showed that memory for two different dimensions (gender of a speaker and language of presentation) of a spoken message were independent of each other. Participants in the gender-relevant condition were told that two males (male English speaker and male Spanish speaker) were litigating against two females (female Spanish speaker and female English speaker) over a piece of land in contention. The other participants in the language-relevant condition were told that two Spanish speaking people (male and 
female) were litigating against two English speaking people (male and female). All participants were then asked to listen to a courtroom hearing tape and decide who was entitled to the piece of land. A subsequent memory test found that gender features were better recalled than language features in the gender-relevant condition, whereas language features were better recalled than gender features in the language-relevant condition. This finding dovetails with the finding by Brewer et al. (1999) also showing that witnesses' description accuracy on a suspect's appearance was not correlated with their accuracy on a suspect's actions.

Overall, the results showing a lack of substantial relationships between object description accuracy or quantity and facial identification accuracy is consistent with the idea that faces are processed differently than non-face objects, as suggested in previous literature (e.g., Curby \& Gauthier, 2007; Reynolds \& Pezdek, 1992). These studies argue that faces are unique because they entail visual processing mechanisms that differ from objects. Hence in the context of the present study, it is logical that there would be little to no association between object description and facial identification for instance. Similarly, we can examine the findings from the perspective of recall and recognition, given that the underlying process for descriptions is recall, whereas the underlying process for identifications is recognition. As Hollingworth (1913) notes, the primary distinction between recall and recognition is that the former requires the retrieval of contextual, specific details of an encounter, whereas the latter depends on a more subjective sense of a previously encountered detail. Thus, recall and recognition seem to rely on cognitive 
processes that are independent of each other, and this can account for the lack of a relationship between description accuracy and identification accuracy.

Nonetheless, the finding of a lack of substantial relationships between description and identification accuracy is practically important from the perspective of an investigator or a juror assessing a witnesses' credibility at trial. From an investigator's perspective, the fact that a witness's accuracy on describing objects from a crime scene is not strongly correlated with his/her identification accuracy indicates that the investigator can pursue a suspect identified by the witness, even though he/she had been previously inaccurate on describing objects present at the crime scene. In essence, the overall usefulness of information provided by a witness would be preserved and investigators could rely on aspects of the witness's account that have not been determined to be inaccurate. For the purposes of assessing a witness's credibility in the courtroom, this finding suggests that jurors should not necessarily discount a witness's subsequent lineup identification based solely on the fact that the witness was shown to have been inaccurate in describing an object relevant to the crime scene. Indeed, educating jurors about the lack of a substantial relationship between description accuracy and identification accuracy could be useful for their assessment of a witness' overall credibility in the courtroom.

The present study also examined the influence of weapon presence on description and identification accuracy. The results showed a weapon focus effect; the presence of a weapon decreased facial identification accuracy and facial description accuracy. This finding is consistent with previous research showing that weapon presence negatively 
affected both identification accuracy and recall accuracy (see Steblay, 1992). Indeed, the weapon focus effect has been consistently demonstrated to be robust across several experimental manipulations. Furthermore, results indicate that the weapon focus effect is not limited to the target holding the weapon; the weapon harmed face identification accuracy even when a different person was holding the weapon. This is one of the very few studies to address this question, as most other studies use only a single perpetrator event.

The presence of a weapon did not however affect either object description accuracy or object identification accuracy. This finding is consistent with the attentional competition framework, in which the eyewitness's attention is first drawn to faces and weapons; when encoding time is limited, attention is thus divided between these features, resulting in a weapon focus effect. But as encoding time increases, attention can be diverted to other objects (in this case a bag and a car). Note that this explanation explains all of the following observed results: (a) the weapon focus effect was found for faces but not objects (since object encoding never competes with weapon encoding); (b) the weapon focus effect was eliminated at long encoding times (since the witness had sufficient time to encode both the faces and the weapon); and (c) increasing encoding time improves recognition of objects but not faces (since faces were primarily encoded immediately).

One of the theoretical explanations for the weapon focus effect - the "unusual item hypothesis." The unusual item hypothesis states that it is more difficult to process scenes that include a weapon or unusual object than scenes that do not. Consequently, the 
weapon would draw significant attentional resources, to the detriment of memory for the other aspects of the scene (Steblay, 1992; Fawcett, Russell, Peace, \& Christie, 2013). An unusual object has been defined in previous weapon focus literature as any object that may be considered unexpected in the context in which it was presented. For example, Pickel (1998) used a whole raw chicken, and found that there was a negative effect on accuracy for witnesses who saw the chicken, compared to those who did not.

Given that the weapon focus effect can be influenced by the amount of attentional resources devoted to encoding particular details, it follows logically that the amount of time a witness is allowed for encoding would play a similarly influential role. Surprisingly, very few studies within the weapon-focus literature have explicitly manipulated encoding time to examine its influence on the weapon focus effect. Exploring the influence of encoding time on the weapon focus effect allows us to (a) test predictions of the attentional competition framework, and (b) examine whether the effect diminishes with an increase in encoding time, which has strong practical implications. The present study's results revealed that the weapon focus effect found for facial accuracy was moderated by encoding time. Supporting the hypothesis, the weapon focus effect occurred when participants viewed the image for 5 seconds, but did not occur when participants viewed the image for 20 seconds. The implication of this finding in the real world is that the duration of a crime or the amount of time available to a witness to encode information during the crime can significantly impact the witness' vulnerability to the weapon focus effect. From a practical perspective, this finding suggests the possibility that a weapon focus effect in real cases diminishes with increased encoding time. 
Another main finding of the present study was that encoding time influenced object description and identification accuracy, but failed to influence facial description accuracy or identification accuracy. This finding contradicts the Curby and Gauthier (2007) study which found a greater increase in visual short term memory (VSTM) capacity for faces than objects as encoding time increased. The authors asserted that more time was needed to encode faces because they are greater in complexity compared to objects. In addition to Curby and Gauthier's study, other studies (see Eng et al., 2006) also found slower visual search rates for faces compared to objects, and asserted that faces placed a greater burden on encoding mechanisms, compared to objects. Therefore, from the perspective of these studies, an increase in encoding time should have had beneficial effects for memory for faces. There is, however, a key difference that may explain the apparent discrepancy between these studies. Earlier studies tested memory for faces and objects separately, and as such there was no attentional competition. In contrast, the present study presented a scene in which both objects and faces were present simultaneously, and witnesses' attention could be drawn by either faces or objects. The present study's attentional competition framework suggests that faces and objects would compete for attentional resources, and it is likely this competition for attentional resources that explains the current findings. In line with previous literature suggesting that people are more oriented towards faces than objects, witnesses' attention was first drawn to the faces, to the detriment of memory for objects especially when encoding time is limited. With adequate encoding time however, people were able to encode objects as well, meaning that an increase in encoding time was more beneficial to memory for objects compared to faces. 
Diagnosticity and information gain analyses revealed that participants recognized objects from lineups at a rate greater than chance, particularly with increased encoding time. This indicates that object identification contains diagnostic value; in fact, the observed informational value of some object identifications was even greater than that contained in suspect identifications. This means that law enforcement officials could possibly derive useful information by asking witnesses to identify crime-related objects such as a car from a lineup, in instances where getting description information is difficult. For instance, a traumatized victim or witness to a crime may have a harder time describing an ordeal in detail, but may be more comfortable pointing out a crime-relevant object from a lineup. Although it is uncommon for law enforcement to administer object lineups, results suggest they may be beneficial if even used merely as a supplementary tool to complement object description accounts provided by a witness. This is a potentially very important finding, and warrants future research.

There are a few limitations with regards to the present study. For instance, although a weapon focus effect was found for overall face identification, it should be noted that this effect was only found for one of the target faces when results were analyzed separately for each target. The exact reason for this finding is unclear. However, a speculative but plausible explanation is that the faces for which the effect was not found may have been less complex compared to the face for which the effect was found, leading to the possibility that it may have been easier to encode those faces. Indeed, as shown in Table 3, the hit rates for the three targets (Male A, Female A, and Female B) were all 
slightly higher than the hit rate for the target for which the weapon focus effect was found (Male B).

Although there is support for the view that the weapon focus effect diminishes with encoding time, this result was found when using only two encoding time periods ( 5 seconds versus 20 seconds). It is consequently difficult to know if the results could be replicated using even longer encoding time periods. The reason why such a line of inquiry may be useful is that there is a possibility that there is a basic time threshold at which a witness can adequately encode details, and thereby diminish the influence of weapon presence. Consequently, beyond that threshold, increasing encoding time may not have much of an effect. Given the paucity of empirical research that explicitly manipulates encoding time, future research is needed to examine the extent to which this finding can be generalized across various encoding time manipulations. This can be ascertained empirically by future research adopting a wide range of encoding time intervals to examine this issue.

Although the results show that object lineups provide diagnostic value, it is difficult to ascertain the extent to which this finding is generalizable. For instance, it is difficult to know whether the usefulness of object lineups generalizes across all types of objects, or whether it is specific to certain types of objects. Additionally, there is for instance no way to determine whether witnesses would perform similarly on object lineups involving objects with which they are familiar, compared to objects with which they have little familiarity. Future research should continue to probe the usefulness and generalizability of objects lineups by examining a wide range of relevant objects. 
Furthermore, we know many manipulations that affect the accuracy of facial identifications (e.g., pre-lineup instructions, filler selection, etc.). A relevant question then is whether these same manipulations affect object identifications as well, and future research may wish to examine this.

In conclusion, the present study has both theoretical and practical implications. Theoretically, the results are generally consistent with the attentional competition framework, a novel model describing how eyewitnesses allocate attentional resources. This model successfully predicted the effects of differing encoding time on memory for faces, objects, and the weapon focus effect. Nonetheless, it failed to account for the lack of an effect of encoding time on description-identification correlations. Future research should further develop this model.

From a practical perspective, the current study's results are useful to law enforcement, judges, attorneys, and jurors. For investigators, knowing that there is a lack of substantial relationship between description accuracy and identification accuracy enables them to resist discounting aspects of a witness' information solely due to prior inaccuracy on another aspect. For jurors and judges, such information provides a framework through which they can evaluate a witness' credibility in the courtroom. Furthermore, the results from the present study shed light on the practical implications of the weapon-focus effect. Knowledge about how the weapon focus effect is influenced by factors such as encoding time for instance enables jurors or judges to make thorough evaluations of testimony in cases involving a weapon. Educating jurors and judges on these issues will enable them to ask critical questions about eyewitness memory when 
assessing witness credibility in the courtroom. Ultimately, this would inure to the benefit of the legal system. 


\section{References}

Biederman, I. (1987). Recognition-by-components: A theory of human image understanding. Psychological Review, 94, 115-147.

Brewer, N., Potter, R., Fisher, R. P., Bond, N. and Luszcz, M. A. (1999), Beliefs and data on the relationship between consistency and accuracy of eyewitness testimony. Applied Cognitive Psychology, 13, 297-313.

Bruce, V, \& Humphreys, G. (1994). Recognizing objects and faces. Visual Cognition, 1, 141-180.

Carey, S. (1992). Becoming a face expert. Philosophical Transactions of the Royal Society of London B: Biological Sciences, 335, 95-103.

Carey, S., \& Diamond, R. (1994). Are faces perceived as configurations more by adults than by children? Visual Cognition, 1, 253-274.

Chance, J. E., \& Goldstein, A. G.. (1976). Recognition of faces and verbal labels. Bulletin of the Psychonomic Society, 7, 384-386.

Charman, S. D., \& Cahill, B. S. (2012). Witnesses' memories for lineup fillers postdict their identification accuracy. Journal of Applied Research in Memory and Cognition, 1, 11-17.

Charman, S. D., \& Wells, G. L. (2007). Eyewitness lineups: Is the appearance-change instruction a good idea? Law and Human Behavior, 31, 3-22.

Curby, K. M., \& Gauthier, I. (2007). A visual short-term memory advantage for faces. Psychonomic Bulletin \& Review, 14, 620-628.

Cutler, B. L., Penrod, S. D., \& Martens, T. K. (1987). The reliability of eyewitness identification: The role of system and estimator variables. Law and Human Behavior, 11(3), 233.

Dial, A. R., \& Ellis, L. (2010). Building a believable case through credible witness testimony. Brief, 39(3), 32-38.

Easterbrook, J. A. (1959). The effect of emotion on cue utilization and the organization of behavior. Psychology Review, 66, 183-201.

Ellis, H.D. (1984). Practical aspects of face memory. In C.L. Wells and E.D. Loftus (Eds) Eyewitness Testimony - Psychological Perspectives. Cambridge: Cambridge University Press. 
Ellis, H. D., Davies, G. M., \& Shepherd, J. W. (1977). Experimental studies of face identification. National Journal of Criminal Defense, 3, 219.

Eng, H. Y., Chen, D., \& Jiang, Y. (2006). Visual working memory for simple and complex visual stimuli. Psychonomic Bulletin and Review, 12, 1127-1133.

Farah, M. J., Tanaka, J. W., \& Drain, H. M. (1995). What causes the face inversion effect? Journal of Experimental Psychology: Human Perception and Performance, 21, 628-634.

Farah, M. J., Wilson, K. D., Drain, M., \& Tanaka, J. N. (1998). What is "special" about face perception? Psychological Review, 105, 482-498.

Fawcett, J., Russell, E., Peace, K., \& Christie, J. (2013). Of guns and geese: a metaanalytic review of the weapon focus literature. Psychology, Crime, \& Law, 19, 35 $-66$.

Finger, K., \& Pezdek, K. (1999). The effect of the cognitive interview on face identification accuracy: Release from verbal overshadowing. Journal of Applied Psychology, 84, 340-348.

Fisher, R. P., \& Cuervo, A. (1983). Memory for physical features of discourse as a function of their relevance. Journal of Experimental Psychology: Learning, Memory, \& Cognition,9, 130-138.

Fisher, R. P., \& Cutler, B. L. (1995). The relation between consistency and accuracy of eyewitness testimony. In G. Davies, S. Lloyd-Bostock, M. McMurran, \& C. Wilson (Eds.), Law and criminal justice: International developments in research and practice. Berlin: De Gruyter.

Fisher, R. P., Vrij, A., \& Leins, D. (2010). Inconsistency as a predictor of memory inaccuracy and lying. In B. S. Cooper, D. Griesel, and M. Ternes (Eds.), Applied issues in investigative interviewing, eyewitness memory, and credibility assessment. New York: Springer.

Freund, R. D., Brelsford, J. W., \& Atkinson, R. C. (1969). Recognition vs. recall: Storage or retrieval differences? Quarterly Journal of Experimental Psychology, 21, 214224.

Gilbert, D.T. (1991). How mental systems believe. American Psychologist, 46, 107-119.

Gilbert, J. A. E., \& Fisher, R. P. (2006). The effects of varied retrieval cues on reminiscence in eyewitness memory. Applied Cognitive Psychology, 20, 723-739.

Goldstein, A. G., \& Chance, J. (1971). Visual recognition memory for complex configurations. Perception \& Psychophysics, 9, 237-241. 
Goldstein, A. G., Johnson, K. S., \& Chance, J. E. (1979). Does fluency of face description imply superior face recognition? Bulletin of the Psychonomic Society, $13,15-18$.

Haxby, J.V., Ungerleider, L.G., Clark, V.P., Schouten, J.L., Hoffman, E.A., \& Martin, A. (1999). The effect of face inversion on activity in human neural systems for face and object perception. Neuron, 22, 189-199.

Hollingworth , H. C. (1913). Characteristic differences between recall and recognition. American Journal of Psychology, 24, 532-544.

Kanwisher, N. , \& Yovel, G. ( 2006 ). The fusiform face area: A cortical region specialized for the perception of faces. Philosophical Transactions of the Royal Society of London. Series B, 361, $2109-2128$.

Johnson, M.H., Dziurawiec, S., Ellis, H., \& Morton, J. (1991). Newborns’ preferential tracking of face-like stimuli and its subsequent decline. Cognition, 40, 1-19.

Leder, H., \& Bruce, V. (2000). When inverted faces are recognized: The role of configural information in face recognition. Quarterly Journal of Experimental Psychology, A, 53(2), 513-536.

Loftus, E. F., Loftus, G. R., \& Messo, J. (1987). Some facts about "weapon focus." Law and Human Behavior, 11(1), 55-62.

Loftus, G. R. (1971). Comparison of recognition and recall in a continuous memory task. Journal of Experimental Psychology, 91, 220-226.

Loftus, G. R., \& Mackworth, N. H. (1978). Cognitive determinants of fixation location during picture viewing. Journal of Experimental Psychology-Human Perception and Performance, 4, 565-572.

Maass, A., \& Köhnken, G. (1989). Eyewitness identification: Simulating the" weapon effect." Law and Human Behavior, 13(4), 397.

McKone, E., \& Greenfell, T. (1999). Orientation invariance in naming rotated objects: individual differences and repetition priming. Perception and Psychophysics, 61, 1590-1603.

McKone, E., Martini, P., \& Nakayama, K. (2001). Categorical perception of face identity in noise isolates configural processing. Journal of Experimental Psychology: Human Perception \& Performance, 27, 573-599. 
McKone, E., \& Robbins, R. (2011). Are faces special? In Calder, A., Rhodes, G., Johnson, M., \& Haxby, J. (Eds.), Oxford handbook of face perception. Oxford, UK: Oxford University Press.

Meissner, C. A., Brigham, J. C., \& Kelley, C. M. (2001). The influence of retrieval processes in verbal overshadowing. Memory \& Cognition, 29, 179-186.

Pascalis, O., \& Kelly, D. (2009). The Origins of Face Processing in Humans: Phylogeny and Ontogeny. Perspectives on Psychological Science, 4(2), 200-209.

Pickel, K. (1998). Unusualness and threat as possible causes of weapon focus. Memory, 6, 277-295.

Pigott, M. A., \& Brigham, J. C. (1985). Relationship between accuracy of prior description and facial recognition. Journal of Applied Psychology, 70, 547-555.

Piggott, M.A., Brigham, J.C., \& Rothwell, R.K. (1990). A field study on the relationship between quality of eyewitness descriptions and identification accuracy. Journal of Police Science and Administration, 17, 84-88.

Porter, S., \& ten Brinke, L. (2009). Dangerous decisions: A theoretical framework for understanding how judges assess credibility in the courtroom. Legal and Criminological Psychology, 14, 119-134.

Pryke, S. Lindsay , R. C. L. Dysart, J. E. Dupuis , P. (2004). Multiple independent identification decisions: a method of calibrating eyewitness identifications. Journal of Applied Psychology, 89, 73-84.

Reed, C. L., Stone, V., Bozova, S., \& Tanaka, J. (2003). The body inversion effect. Psychological Science, 14, 302-308.

Reynolds, J. K., \& Pezdek, K. (1992). Face recognition memory: The effect of exposure duration and encoding instructions. Applied Cognitive Psychology, 6, 279-292.

Richler, J. J., Cheung, O. S., \& Gauthier, I. (2011). Holistic processing predicts face recognition. Psychological Science, 22, 464-471.

Robbins, R., \& McKone, E. (2003). Can holistic processing develop with practice? Cognition, 81(1), 79-107.

Shapiro, P. N., \& Penrod, S. (1986). Meta-analysis of facial identification studies. Psychological Bulletin, 100, 139-156.

Shepherd, J.W. \& Ellis, H.D. (1996). Face recall-methods and problems. In S.L. Sporer, R.S. Malpass and G. Köhnken (Eds), Psychological Issues in Eyewitness Identification. Hillsdale, NJ: Lawrence Erlbaurn. 
Steblay, N. (1992). A meta-analytic review of the weapon-focus effect. Law and Human Behavior, 16, 413-424.

Tanaka, J. W., \& Farah, M. J. (1993). Parts and wholes in face recognition. Quarterly Journal of Experimental Psychology, 46A, 225-245.

Tooley, V., Brigham, J. C., Maass, A., \& Bothwell, R. K. (1987). Facial Recognition: Weapon Effect and Attentional Focus. Journal of Applied Social Psychology, $17(10), 845-859$.

Tovee, M. J. (1998). Is Face Processing Special? Neuron, 21, 1239-1242.

Tversky, B. (1973). Encoding processes in recognition and recall. Cognitive Psychology, $5,275-287$.

Tversky, B. \& Sherman, T. (1975). Picture memory improves with longer on time and off time. Journal of Experimental Psychology: Human Learning and Memory, 1, 114-118.

Wells, G. L. (1985). Verbal descriptions of faces from memory: Are they diagnostic of identification accuracy? Journal of Applied Psychology, 70, 619-626.

Wells, G.L. (1993). What do we know about eyewitness identification? American Psychologist, 48, 553-571.

Wells, G. L., \& Lindsay, R. C. L. (1980). On estimating the diagnosticity of eyewitness nonidentifications. Psychological Bulletin, 88, 776-784.

Wells, G. L., \& Olson, E. A. (2002). Eyewitness identification: Information gain from incriminating and exonerating behaviors. Journal of Experimental Psychology: Applied, 8, 155-167.

Wells, G. L., \& Windschitl, P. D. (1999). Stimulus sampling and social psychological experimentation. Personality and Social Psychology Bulletin, 25, 1115-1125.

Wheeler, M. E., \& Treisman, A. M. (2002). Binding in short-term visual memory. Journal of Experimental Psychology: General, 131, 48-64.

Wolfskiel, M. P., \& Brigham, J. C. (1985). The relationship between accuracy of prior description and facial recognition. Journal of Applied Psychology, 70.

Yarmey, A. D. (1971). Recognition memory for familiar "public" faces: Effects of orientation and delay. Psychonomic Science, 24, 286-288. 
Yerkes, R. M., \& Dodson, J. D. (1908). The relation of strength of stimulus to rapidity of habit-formation. Journal of comparative neurology and psychology, 18(5), 459482.

Yin, R. K. (1969). Looking at upside-down faces. Journal of Experimental Psychology: Human Learning and Memory, 7, 181-190.

Young, A. W., Hellawell, D., \& Hay, D. C. (1987). Configurational information in face perception. Perception, 16(6), 747-759. 
Table 1

Target-Present and Target-Absent Identification Accuracy Proportions for Facial identifications, Object identifications, Facial descriptions, and Object descriptions as a function of Encoding Time.

$\underline{\text { Encoding Time }}$

5 Seconds 20 Seconds

Target-Present

$\begin{array}{lcc}\text { Facial identifications } & 0.42 & 0.46 \\ \text { Object identifications } & 0.51 & 0.71^{* *}\end{array}$

Target-Absent

Facial identifications

0.49

0.56

Object identifications

0.56

$0.68^{* *}$

Descriptions

Facial descriptions

0.72

0.74

Object descriptions

0.69

$0.74 * *$

Note: $* * p<.01$ 
Table 2

Bivariate Correlations between Identification Accuracy and Overall Description Accuracy, Facial Description Accuracy, Object Description Accuracy, Overall Description Quantity, Facial Description Quantity, and Object Description Quantity for Target-present and Target-absent Lineups.

Target-Present Identification Accuracy
Target-Absent Identification Accuracy

Overall description accuracy

$0.15 * *$

$-0.01$

Facial description accuracy

0.08

0.02

Object description accuracy

$0.09 *$

0.04

Overall description quantity

$0.13^{*}$

$0.10 *$

Facial description quantity

0.05

0.07

Object description quantity

0.03

$-0.08$

Notes: ${ }^{*} \mathrm{p}<.05, * * \mathrm{p}<.01$ 
Table 3

Identification Accuracy Proportions of Specific Targets across Weapon Presence, Encoding Time and Lineup Type Conditions.

\begin{tabular}{|c|c|c|c|c|c|c|}
\hline & \multicolumn{2}{|c|}{ Weapon } & \multicolumn{2}{|c|}{ No Weapon } & \multicolumn{2}{|c|}{ Overall } \\
\hline & $\mathrm{TP}$ & TA & $\mathrm{TP}$ & $\mathrm{TA}$ & $\mathrm{TP}$ & TA \\
\hline \multicolumn{7}{|c|}{5 seconds condition } \\
\hline Male A & 0.33 & 0.50 & 0.48 & 0.60 & 0.40 & 0.55 \\
\hline Female A & 0.48 & 0.48 & 0.47 & 0.43 & 0.47 & 0.46 \\
\hline Car A & 0.51 & 0.53 & 0.47 & 0.59 & 0.49 & 0.56 \\
\hline Bag A & 0.28 & 0.43 & 0.24 & 0.39 & 0.26 & 0.41 \\
\hline Male B & 0.22 & 0.47 & 0.50 & 0.49 & 0.35 & 0.48 \\
\hline Female B & 0.37 & 0.31 & 0.45 & 0.54 & 0.41 & 0.42 \\
\hline Car B & 0.60 & 0.63 & 0.54 & 0.64 & 0.57 & 0.63 \\
\hline Bag B & 0.28 & 0.40 & 0.26 & 0.48 & 0.27 & 0.44 \\
\hline \multicolumn{7}{|c|}{20 seconds condition } \\
\hline Male A & 0.42 & 0.72 & 0.49 & 0.74 & 0.46 & 0.73 \\
\hline Female A & 0.52 & 0.51 & 0.57 & 0.51 & 0.55 & 0.51 \\
\hline Car A & 0.61 & 0.68 & 0.75 & 0.65 & 0.68 & 0.67 \\
\hline Bag A & 0.43 & 0.61 & 0.45 & 0.60 & 0.44 & 0.61 \\
\hline Male B & 0.41 & 0.50 & 0.43 & 0.57 & 0.42 & 0.53 \\
\hline Female B & 0.43 & 0.60 & 0.42 & 0.35 & 0.43 & 0.47 \\
\hline Car B & 0.83 & 0.65 & 0.81 & 0.69 & 0.82 & 0.67 \\
\hline Bag B & 0.51 & 0.56 & 0.41 & 0.61 & 0.46 & 0.58 \\
\hline \multicolumn{7}{|c|}{ Overall } \\
\hline Male A & 0.37 & 0.61 & 0.48 & 0.67 & 0.43 & 0.64 \\
\hline Female A & 0.50 & 0.50 & 0.52 & 0.47 & 0.51 & 0.49 \\
\hline Car A & 0.56 & 0.52 & 0.61 & 0.62 & 0.59 & 0.62 \\
\hline Bag A & 0.36 & 0.52 & 0.35 & 0.50 & 0.36 & 0.51 \\
\hline Male B & 0.31 & 0.48 & 0.47 & 0.53 & 0.39 & 0.51 \\
\hline Female B & 0.40 & 0.41 & 0.44 & 0.45 & 0.42 & 0.45 \\
\hline Car B & 0.72 & 0.64 & 0.68 & 0.67 & 0.70 & 0.65 \\
\hline Bag B & 0.40 & 0.48 & 0.34 & 0.55 & 0.37 & 0.51 \\
\hline
\end{tabular}


Table 4

Diagnosticity Estimates for each Target as a Function of Encoding Time

\begin{tabular}{lccc} 
& 5 seconds & 20 seconds & Difference (sig.) \\
Male A & 3.30 & 9.20 & $z=1.90(p=.03)^{*}$ \\
Female A & 3.92 & 5.50 & $z=1.15(p=.13)$ \\
Car A & 4.45 & 11.33 & $z=2.90(p=.00)^{* *}$ \\
Bag A & 5.20 & 11.00 & $z=2.15(p=.02)^{*}$ \\
Weapon A & 1.30 & 2.03 & $z=1.30(p=.10)$ \\
Male B & 3.18 & 3.23 & $z=0.40(p=.34)$ \\
Female B & 8.20 & 10.75 & $z=0.50(p=.31)$ \\
Car B & 7.13 & 11.71 & $z=3.00(p=.00)^{* *}$ \\
Bag B & 1.13 & 2.30 & $z=2.45(p=.01)^{* *}$ \\
Weapon B & 1.11 & 1.19 & $z=0.33(p=.37)$ \\
\hline
\end{tabular}

Notes: $* \mathrm{p}<.05, * * \mathrm{p}<.01$ 


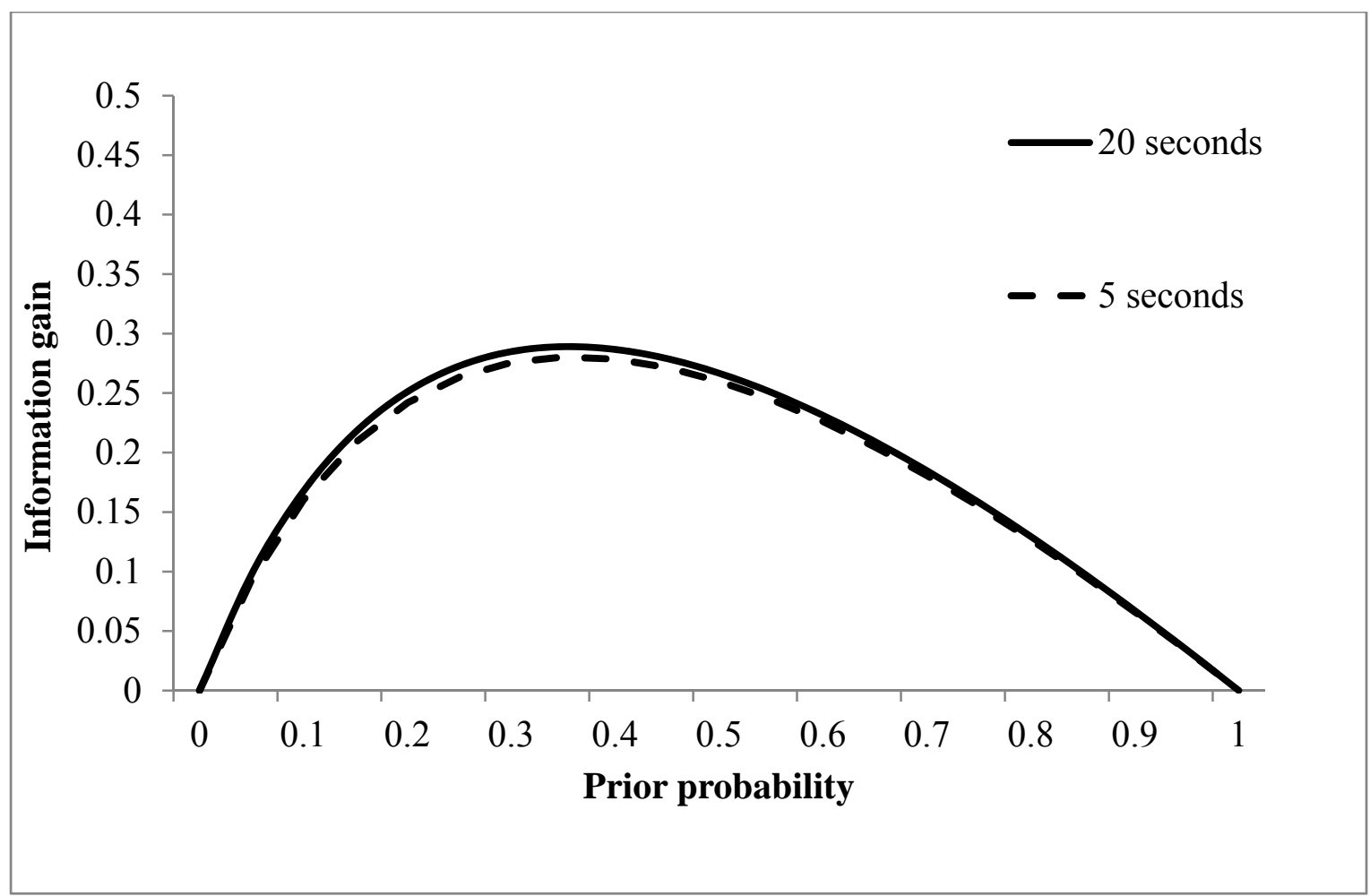

Figure 1. Information gain from identification of male suspect for when encoding time is 5 seconds versus when encoding time is 20 seconds. 


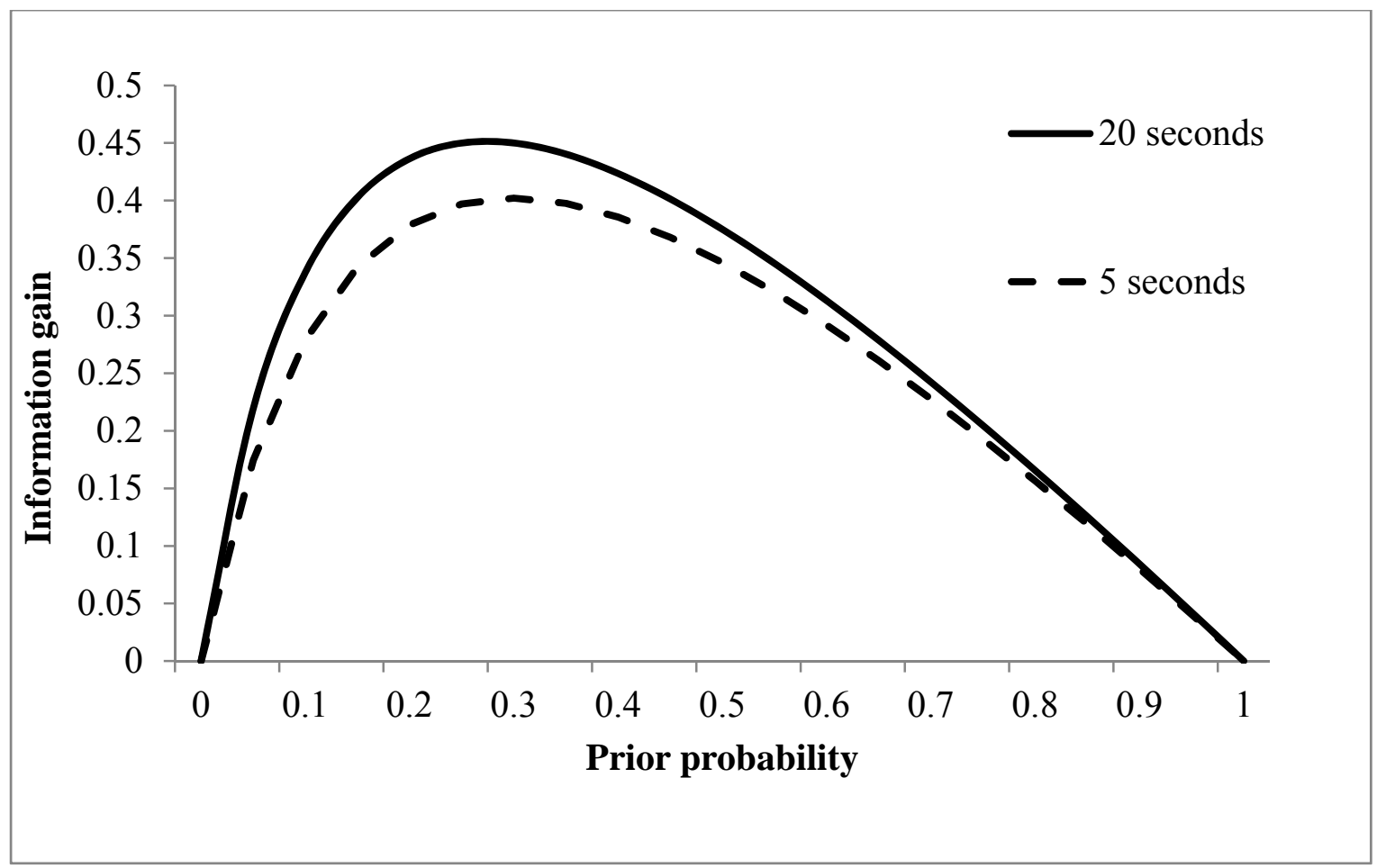

Figure 2. Information gain from identification of female suspect for when encoding time is 5 seconds versus when encoding time is 20 seconds. 


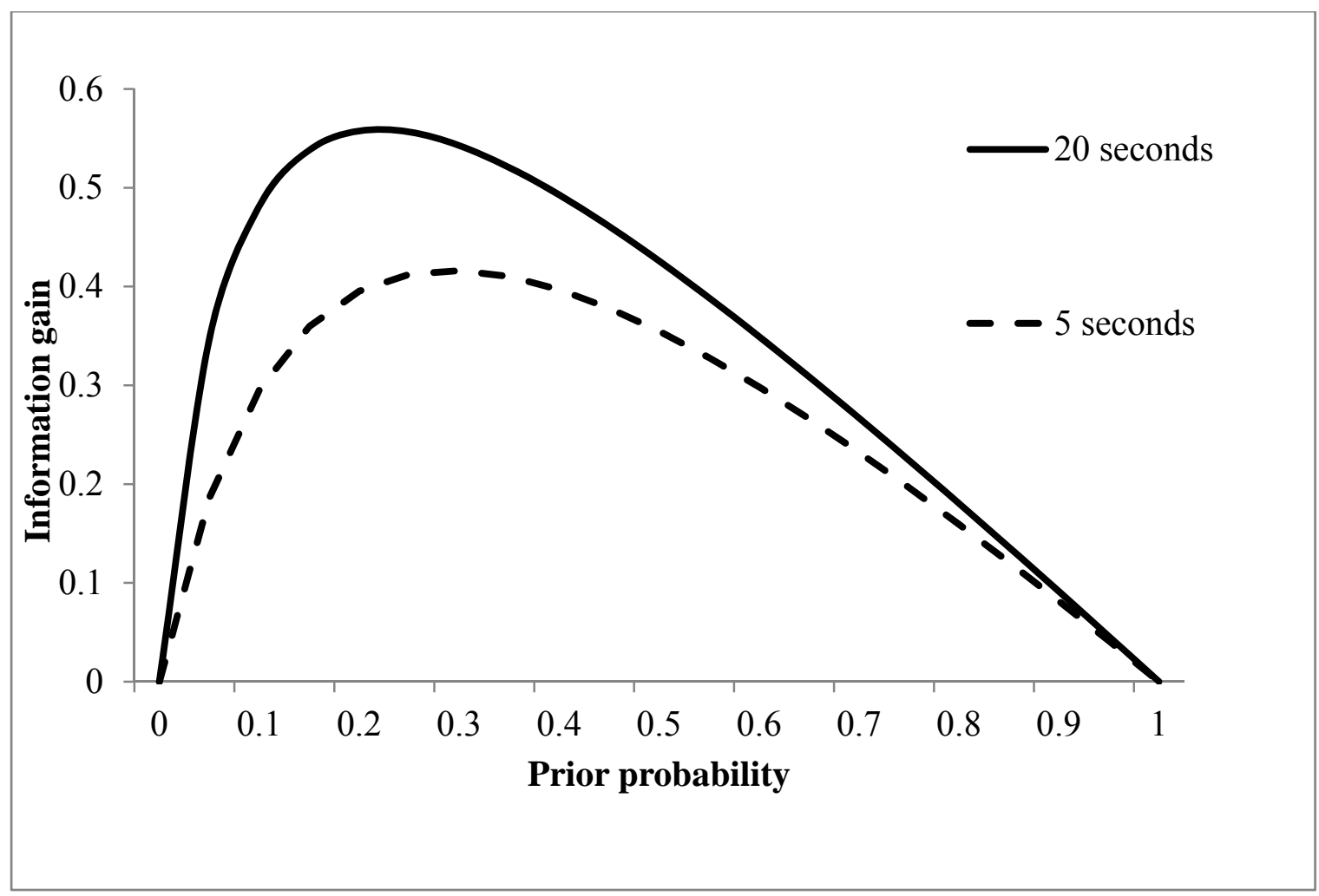

Figure 3. Information gain from identification of a target car for when encoding time is 5 seconds versus when encoding time is 20 seconds. 


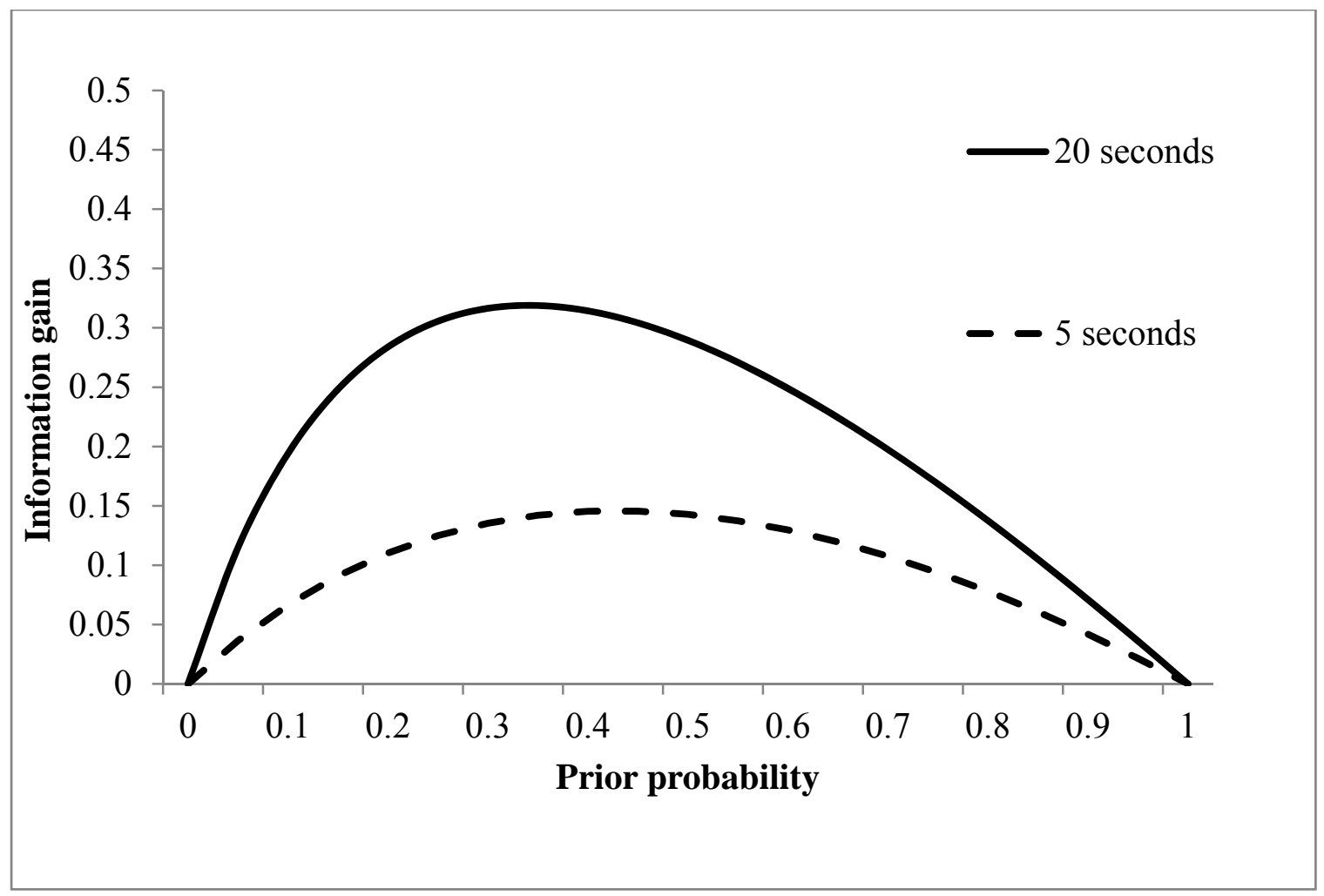

Figure 4. Information gain from identification of a target bag for when encoding time is 5 seconds versus when encoding time is 20 seconds. 


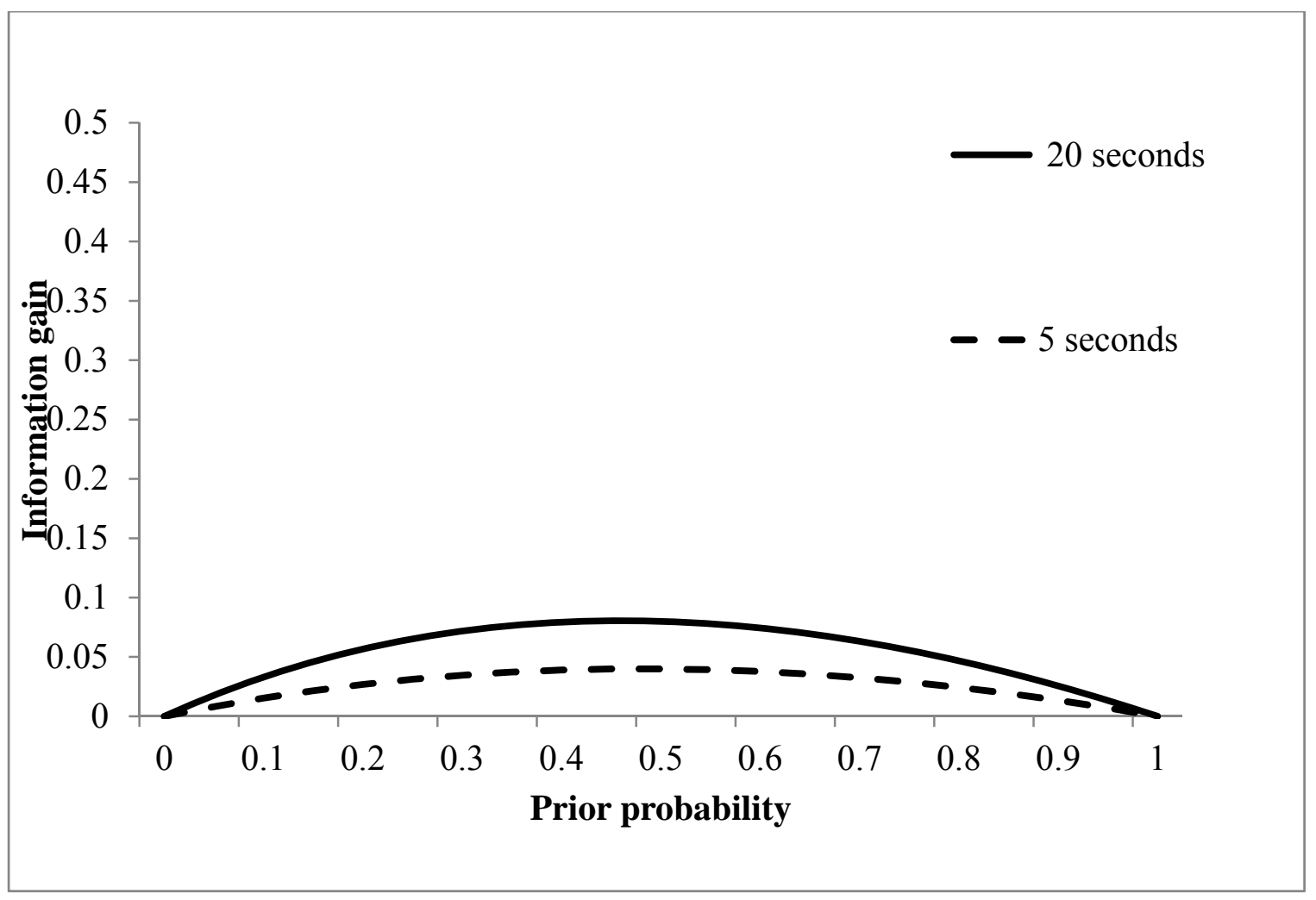

Figure 5. Information gain from identification of a target weapon for when encoding time is 5 seconds versus when encoding time is 20 seconds. 
VITA

\title{
SEYRAM MAWUKO KEKESSIE
}

\author{
Born, Accra, Ghana
}

2005-2009

B.A., Psychology

Hanover College

Hanover, Indiana

2008

Save Darfur Coalition, Washington DC

2009-2015

Teaching Assistant

Florida International University

Miami, Florida

2013

M.S., Psychology

Florida International University

Miami, Florida

$2013 \& 2015$

APLS Travel Award Recipient

2013-2015

Doctoral Candidate

Florida International University

Miami, Florida

\section{PRESENTATIONS}

Kekessie, S., \& Charman, S. (2014). The Influence of the 'Not Sure' Option on Eyewitness Lineup Performance. Presented at the American Psychology and Law Society Conference, Portland, Oregon.

Kekessie, S., Charman, S. \& Carbone, J.N. (2012)Coherence in Legal Decision-Making: The Moderating Effect of Evidence Order. Presented at the American Psychology and Law Society Conference, San Juan, Puerto Rico. 